\title{
ON THE IRREDUCIBILITY OF THE EXTENSIONS OF BURAU AND GASSNER REPRESENTATIONS
}

\author{
MOHAMAD N. NASSER AND MOHAMMAD N. ABDULRAHIM
}

\begin{abstract}
Let $C b_{n}$ be the group of basis conjugating automorphisms of a free group $\mathbb{F}_{n}$, and $C_{n}$ the group of conjugating automorphisms of $\mathbb{F}_{n}$. Valerij G. Bardakov has constructed representations of $C b_{n}, C_{n}$ in the groups $G L_{n}\left(\mathbb{Z}\left[t_{1}^{ \pm 1}, \ldots, t_{n}^{ \pm 1}\right]\right)$ and in $G L_{n}\left(\mathbb{Z}\left[t^{ \pm 1}\right]\right)$ respectively, where $t_{1}, \ldots, t_{n}, t$ are indeterminate variables. We show that these representations are reducible and we determine the irreducible components of the representations in $G L_{n}(\mathbb{C})$, which are obtained by giving values to the variables above. Next, we consider the tensor product of the representations of $C b_{n}, C_{n}$ and study their irreduciblity in the case $n=3$.
\end{abstract}

II

\section{INTRODUCTION}

The braid group on $n$ strings, $B_{n}$, is the abstract group with generators $\sigma_{1}, \ldots, \sigma_{n-1}$ and a presentation as follows:

$$
\begin{array}{ll}
\sigma_{i} \sigma_{i+1} \sigma_{i}=\sigma_{i+1} \sigma_{i} \sigma_{i+1}, & i=1,2, \ldots, n-2, \\
\sigma_{i} \sigma_{j}=\sigma_{j} \sigma_{i}, & |i-j|>2 .
\end{array}
$$

The pure braid group, $P_{n}$, is defined as the kernel of the homomorphism $B_{n} \mapsto S_{n}$ defined by $\sigma_{i} \mapsto(i \quad i+1), 1 \leq i \leq n-1$, where $S_{n}$ is the symmetric group of $n$ elements.

The most famous linear representation of $B_{n}$ is Burau representation [4], and the most famous linear representation of $P_{n}$ is Gassner representation [3].

One of the generalizations of the braid group $B_{n}$ is the group $C_{n}$ of conjugating automorphisms of $\mathbb{F}_{n}$, the free group of rank $n$ with the generators $x_{1}, \ldots, x_{n}$ (see $[6])$. Here $C_{n}$ is defined to be the subgroup of $A u t\left(\mathbb{F}_{n}\right)$ that satisfies for any $\phi \in C_{n}$, $\phi\left(x_{i}\right)=f_{i}^{-1} x_{\Pi(i)} f_{i}$, where $\Pi$ is a permutation on $\{1,2, \ldots, n\}$ and $f_{i}$ lies in $\mathbb{F}_{n}$. By the Theorem of Artin [3], the group $B_{n}$ admits a faithful representation in $A u t\left(\mathbb{F}_{n}\right)$ such that an automorphism $\beta$ satisfies the following two conditions:

(1) $\beta\left(x_{i}\right)=f_{i}^{-1} x_{\Pi(i)} f_{i}, \quad 1 \leq i \leq n$,

(2) $\beta\left(x_{1} x_{2} \ldots x_{n}\right)=x_{1} x_{2} \ldots x_{n}$,

where $\Pi$ is a permutation on $\{1,2, \ldots, n\}$ and $f_{i} \in \mathbb{F}_{n}$. Recall that condition (1) is the defining condition for an automorphism of $\mathbb{F}_{n}$ to be in $C_{n}$, the group of conjugating automorphisms.

Key words and phrases. Braid group, Free group, Magnus representation, Burau representation, Gassner representation.

Mathematics Subject Classification. Primary: 20F36. 
Also, one of the generalizations of the pure braid group $P_{n}$ is the group of basis conjugating automorphisms $C b_{n}$ [5], which is the subgroup of $C_{n}$ that satisfies for any $\phi \in C b_{n}, \phi\left(x_{i}\right)=f_{i}^{-1} x_{i} f_{i}$, where $f_{i} \in \mathbb{F}_{n}$.

$P_{n}$ is a normal subgroup of $B_{n}$ and $C b_{n}$ is a normal subgroup of $C_{n}$. In addition, the quotient groups $B_{n} / P_{n}$ and $C_{n} / C b_{n}$ are isomorphic to $S_{n}$. A. G. Savuschkina [6] proved that $C_{n}$ is a semidirect product $C_{n}=C b_{n} \lambda S_{n}$.

Denote $\mathbb{F}_{n}^{\prime}=\left[\mathbb{F}_{n}, \mathbb{F}_{n}\right]$, the commutator subgroup of $\mathbb{F}_{n}$, and $\mathbb{A}_{n}=\mathbb{F}_{n} / \mathbb{F}_{n}^{\prime}$. The natural map from $A u t\left(\mathbb{F}_{n}\right)$ into $A u t\left(\mathbb{A}_{n}\right)$ is an epimorphism. The kernel of this map is the group of IA-automorphisms denoted by $I A\left(\mathbb{F}_{n}\right)$ (see [1]).

We consider $C b_{n}$ as a subgroup of $I A\left(\mathbb{F}_{n}\right)$, the group of IA-automorphisms of the group $\mathbb{F}_{n}$.

In [2], Bardakov uses Magnus representation defined in [3, Ch. 3] to construct a linear representation $\rho: I A\left(\mathbb{F}_{n}\right) \mapsto G L_{n}\left(\mathbb{Z}\left[t_{1}^{ \pm 1}, \ldots, t_{n}^{ \pm 1}\right]\right)$. Restricting the representation $\rho$ to $C b_{n}$ we obtain a representation $\hat{\rho}_{G}$, which is an extension of Gassner representation of $P_{n}$. Putting $t_{1}=\ldots=t_{n}$ in the representation $\hat{\rho}_{G}$, we obtain a representation $\hat{\rho}_{B}$ of $C_{n}$, which is an extension of Burau representation of $B_{n}$.

We study, in section 3 , the irreducibility of the representation $\hat{\rho}_{G}$. We prove that $\hat{\rho}_{G}$ is reducible (Theorem 3 ). In order not to get a one-dimensional representation, we assume that one of the $t_{i}$ 's not one. Without loss of generality, we set $t_{n} \neq 1$. We prove that the complex specialization of its $(n-1)$ th degree composition factor $\hat{\phi}_{G}$ is irreducible if and only if $t_{i} \neq 1$ for all $1 \leq i<n$ (Theorem 4 ).

Similarly, we study in section 4 the irreducibility of the representation $\hat{\rho}_{B}$. We prove that $\hat{\rho}_{B}$ is reducible (Theorem 6 ). Also we prove that the complex specialization of its $(n-1)$ th degree composition factor $\hat{\phi}_{B}$ is irreducible (Theorem 7 ).

In section 5 , we prove, for $n=3$, that the tensor product representation $\hat{\phi}_{G}\left(t_{1}, t_{2}, t_{3}\right) \otimes$ $\hat{\phi}_{G}\left(m_{1}, m_{2}, m_{3}\right)$ is irreducible if and only if $\left(t_{1}, t_{2}, t_{3}\right)$ and $\left(m_{1}, m_{2}, m_{3}\right)$ are distinct vectors (Theorem 8 ).

In section 6 , we prove, for $n=3$, that the tensor product representation $\hat{\phi}_{B}(t) \otimes$ $\hat{\phi}_{B}(m)$ is irreducible if and only if $t \neq m$ (Theorem 9 ).

\section{Preliminaries}

The group of conjugating automorphisms, $C_{n}$, is the subgroup of $A u t\left(\mathbb{F}_{n}\right)$ that satisfies for any $\phi \in C_{n}, \phi\left(x_{i}\right)=f_{i}^{-1} x_{\Pi(i)} f_{i}$, where $\Pi$ is a permutation on $\{1,2, \ldots, n\}$ and $f_{i} \in \mathbb{F}_{n}$.

The group of basis conjugating automorphisms, $C b_{n}$, is the subgroup of $C_{n}$ that satisfies for any $\phi \in C b_{n}, \phi\left(x_{i}\right)=f_{i}^{-1} x_{i} f_{i}$, where $f_{i} \in \mathbb{F}_{n}$.

J. McCool [5] proved that the group $C b_{n}$ is generated by the automorphisms

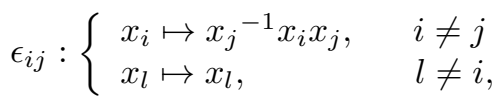

where $1 \leq i \neq j \leq n$.

Recall that $I A\left(\mathbb{F}_{n}\right)$ is generated by the automorphisms $\epsilon_{i j}, 1 \leq i \neq j \leq n$ and the automorphisms

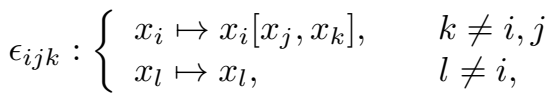

where $[a, b]=a^{-1} b^{-1} a b[2]$. 
In [6], we have $C_{n}=C b_{n} \lambda S_{n}$. This means that $C_{n}$ is generated by the automorphisms $\epsilon_{i j}$, where $1 \leq i \neq j \leq n$, and the permutations $\alpha_{i}$ where $1 \leq i \leq n-1$. Here $\alpha_{i}$ is defined as follows:

$$
\alpha_{i}= \begin{cases}x_{i} \mapsto x_{i+1} & \\ x_{i+1} \mapsto x_{i}, & i=1,2, \ldots, n-1 \\ x_{j} \mapsto x_{j}, & j \neq i, i+1\end{cases}
$$

Definition 1. [2] The group $I A\left(\mathbb{F}_{n}\right)$ is the group of the IA-automorphisms of the group $\mathbb{F}_{n}$. We introduce the representation $\rho: I A\left(\mathbb{F}_{n}\right) \mapsto G L_{n}\left(\mathbb{Z}\left[t_{1}^{ \pm 1}, \ldots, t_{n}{ }^{ \pm 1}\right]\right)$ as follows:

$$
\begin{aligned}
& \epsilon_{i j} \mapsto \rho\left(\epsilon_{i j}\right):\left\{\begin{array}{l}
e_{i} \rho\left(\epsilon_{i j}\right)=t_{j}^{-1}\left(t_{i}-1\right) e_{j}+t_{j}{ }^{-1} e_{i}, \\
e_{l} \rho\left(\epsilon_{i j}\right)=e_{l}, \quad l \neq i,
\end{array}\right. \\
& \epsilon_{i j k} \mapsto \rho\left(\epsilon_{i j k}\right):\left\{\begin{array}{l}
e_{i} \rho\left(\epsilon_{i j k}\right)=e_{i}+t_{i} t_{j}^{-1}\left(t_{k}^{-1}-1\right) e_{j}+t_{i} t_{k}^{-1}\left(1-t_{j}^{-1}\right) e_{k}, \\
e_{l} \rho\left(\epsilon_{i j k}\right)=e_{l}, \quad l \neq i .
\end{array}\right.
\end{aligned}
$$

Here we consider the matrices $\rho\left(\epsilon_{i j}\right)$ and $\rho\left(\epsilon_{i j k}\right)$ as automorphisms of $W_{n}$, a free left $R$-module with basis $\left\{e_{1}, e_{2}, \ldots, e_{n}\right\}$, where $R=\mathbb{Z}\left[t_{1}^{ \pm 1}, \ldots, t_{n}^{ \pm 1}\right]$. Throughout our work, we consider $G L_{n}(R)$ as acting from the left on column vectors and acting from the right on row vectors.

\section{The irreducibility of The REPRESEntations $\hat{\rho}_{G}$}

Definition 2. [2] The representation $\hat{\rho}_{G}$ is defined by

$$
\begin{aligned}
& \hat{\rho}_{G}: C b_{n} \mapsto G L_{n}\left(\mathbb{Z}\left[t_{1}^{ \pm 1}, \ldots, t_{n}^{ \pm 1}\right]\right)
\end{aligned}
$$

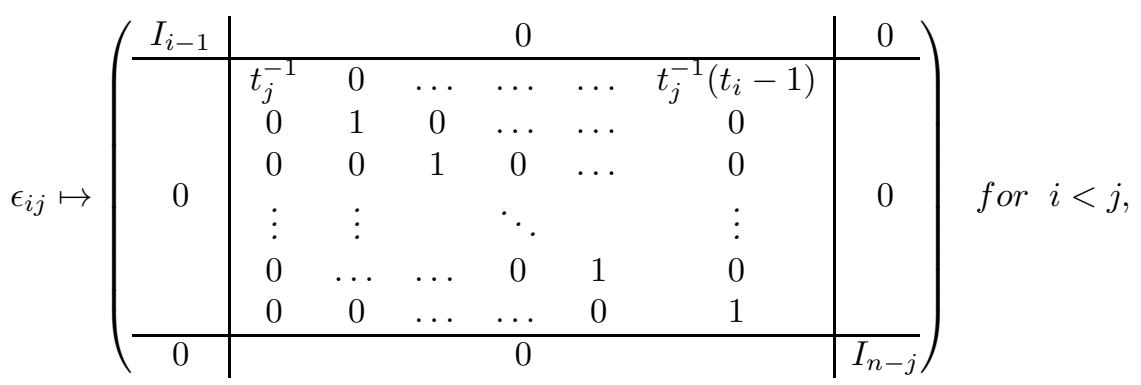

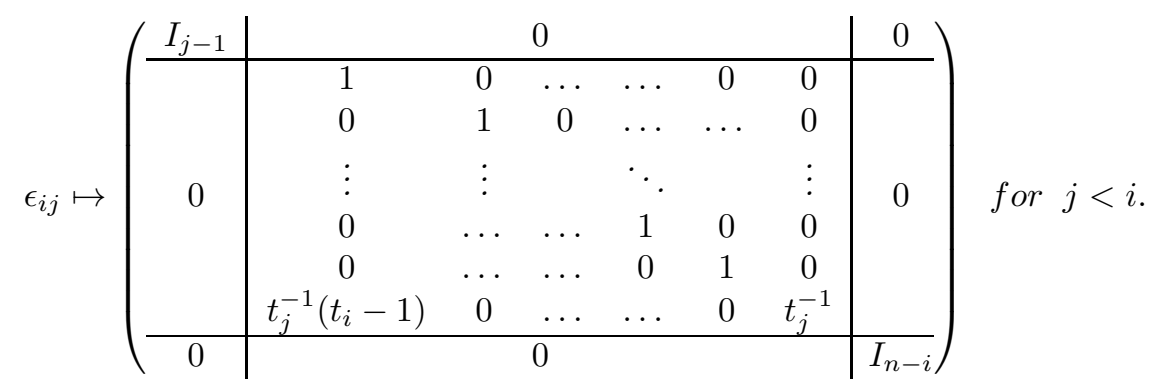

Note that in the cases $i=1$ and $j=1$ we omit the first $i-1$ (respectively $j-1$ ) rows and $i-1$ (respectively $j-1$ ) columns. And in the cases $i=n$ and $j=n$ we omit the last $n-i$ (respectively $n-j$ ) rows and $n-i$ (respectively $n-j$ ) columns.

Theorem 3. The representation $\hat{\rho}_{G}$ is reducible. 
Proof. Let $v=\left[t_{1}-1, t_{2}-1, \ldots, t_{n}-1\right]^{T}$, where $T$ is the transpose. We see that $\epsilon_{i j}(v)=v$ for all $1 \leq i, j \leq n$, and so $v$ is fixed under the generators of $\hat{\rho}_{G}$. Thus $\hat{\rho}_{G}$ is reducible.

We specialize $t_{1}, \ldots, t_{n}$ to non-zero complex numbers. We want to find a composition factor of degree $n-1$ of $\hat{\rho}_{G}$. We may assume, in order not to get a one-dimensional representation, that not all $t_{i}$ 's take on the value one. This means that there exists $t_{j} \neq 1$ for $1 \leq j \leq n$. For the complex vector space $\mathbb{C}^{n}$ of dimension $n$, we consider the basis $S=\{e_{1}, \ldots, e_{j-1}, \underbrace{e_{j+1}, \ldots, e_{n}}_{n-j}, v\}$, where $v=\left[t_{1}-1, t_{2}-1, \ldots, t_{n}-1\right]^{T}$. It is clear that $S$ is a basis of $\mathbb{C}^{n}$ as $t_{j} \neq 1$. Now, to make calculations easier, we assume, without loss of generality, that $j=n$ and so $t_{n} \neq 1$. In this way, the basis $S$ is $\left\{e_{1}, \ldots, e_{n-1}, v\right\}$.

For $i<j \neq n$ :

$\epsilon_{i j}\left(e_{1}\right)=e_{1}, \epsilon_{i j}\left(e_{2}\right)=e_{2}, \ldots, \epsilon_{i j}\left(e_{i-1}\right)=e_{i-1}, \epsilon_{i j}\left(e_{i}\right)=t_{j}^{-1} e_{i}, \epsilon_{i j}\left(e_{i+1}\right)=e_{i+1}$, $\ldots, \epsilon_{i j}\left(e_{j-1}\right)=e_{j-1}, \epsilon_{i j}\left(e_{j}\right)=t_{j}^{-1}\left(t_{i}-1\right) e_{i}+e_{j}, \epsilon_{i j}\left(e_{j+1}\right)=e_{j+1}, \ldots, \epsilon_{i j}\left(e_{n-1}\right)=$ $e_{n-1}, \epsilon_{i j}(v)=v$.

For $j=n$ :

$\epsilon_{i n}\left(e_{1}\right)=e_{1}, \epsilon_{i n}\left(e_{2}\right)=e_{2}, \ldots, \epsilon_{i n}\left(e_{i-1}\right)=e_{i-1}, \epsilon_{i n}\left(e_{i}\right)=t_{n}^{-1} e_{i}, \epsilon_{i n}\left(e_{i+1}\right)=e_{i+1}$, $\ldots, \epsilon_{i n}\left(e_{n-1}\right)=e_{n-1}, \epsilon_{i n}(v)=v$.

For $j<i \neq n$ :

$\epsilon_{i j}\left(e_{1}\right)=e_{1}, \epsilon_{i j}\left(e_{2}\right)=e_{2}, \ldots, \epsilon_{i j}\left(e_{j-1}\right)=e_{j-1}, \epsilon_{i j}\left(e_{j}\right)=e_{j}+t_{j}^{-1}\left(t_{i}-1\right) e_{i}$, $\epsilon_{i j}\left(e_{j+1}\right)=e_{j+1}, \ldots, \epsilon_{i j}\left(e_{i-1}\right)=e_{i-1}, \epsilon_{i j}\left(e_{i}\right)=t_{j}^{-1} e_{i}, \epsilon_{i j}\left(e_{i+1}\right)=e_{i+1}, \ldots$, $\epsilon_{i j}\left(e_{n-1}\right)=e_{n-1}, \epsilon_{i j}(v)=v$.

For $i=n:$

$\epsilon_{n j}\left(e_{1}\right)=e_{1}, \epsilon_{n j}\left(e_{2}\right)=e_{2}, \ldots, \epsilon_{n j}\left(e_{j-1}\right)=e_{j-1}, \epsilon_{n j}\left(e_{j}\right)=-t_{j}^{-1}\left(t_{1}-1\right) e_{1}-t_{j}^{-1}\left(t_{2}-\right.$ 1) $e_{2}-\ldots-t_{j}^{-1}\left(t_{j-1}-1\right) e_{j-1}+t_{j}^{-1} e_{j}-t_{j}^{-1}\left(t_{j+1}-1\right) e_{j+1}-\ldots-t_{j}^{-1}\left(t_{n-1}-1\right) e_{n-1}+t_{j}^{-1} v$, $\epsilon_{n j}\left(e_{j+1}\right)=e_{j+1}, \ldots, \epsilon_{n j}\left(e_{n-1}\right)=e_{n-1}, \epsilon_{n j}(v)=v$.

So, the representation $\hat{\rho}_{G}$, in the new basis $S$, becomes

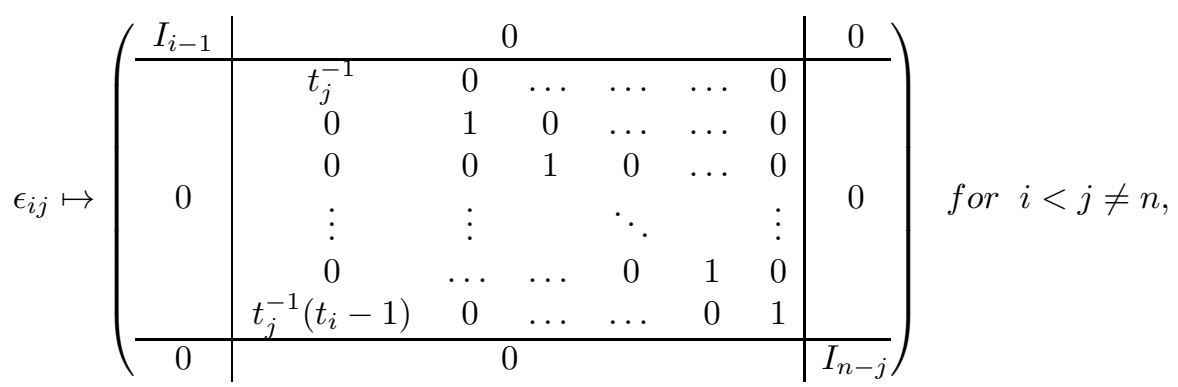

$$
\epsilon_{i n} \mapsto\left(\begin{array}{c|c|c}
I_{i-1} & 0 & 0 \\
\hline 0 & t_{n}^{-1} & 0 \\
\hline 0 & 0 & I_{n-i}
\end{array}\right),
$$




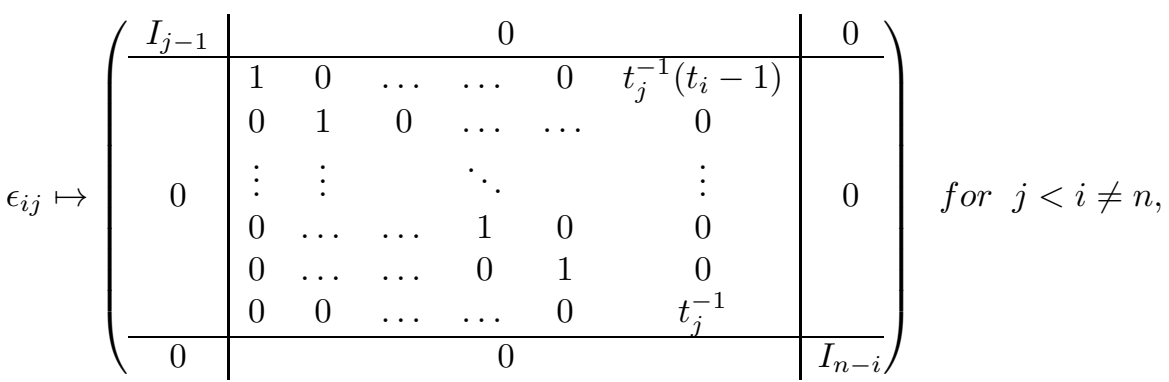

$$
\begin{aligned}
& \epsilon_{n j} \mapsto\left(\begin{array}{ccc|ccccc}
\multicolumn{3}{c|}{I_{j-1}} & \multicolumn{5}{c}{0} \\
\hline q_{1} & \ldots & q_{j-1} & t_{j}^{-1} & q_{j+1} & \ldots & q_{n-1} & t_{j}^{-1} \\
0 & 0 & 0 & 0 & 1 & 0 & \ldots & 0 \\
\vdots & \vdots & \vdots & \vdots & & \ddots & & \vdots \\
0 & 0 & 0 & 0 & \ldots & 0 & 1 & 0 \\
0 & 0 & 0 & 0 & \ldots & \ldots & 0 & 1
\end{array}\right),
\end{aligned}
$$

where $q_{k}=-t_{j}^{-1}\left(t_{k}-1\right)$ for all $1 \leq k \neq j \leq n-1$.

Now, we remove the last row and the last column to obtain the $n-1$ composition factor $\hat{\phi}_{G}$ given by the following generators

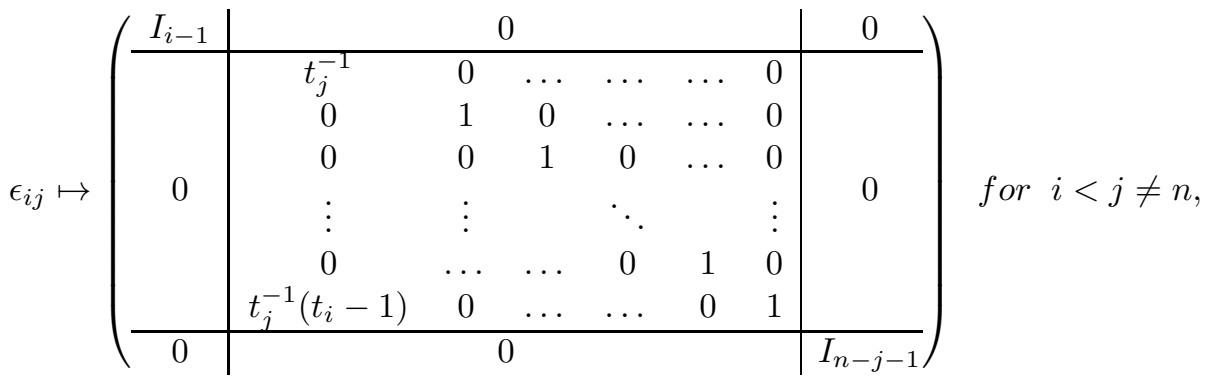

\begin{tabular}{|c|c|c|c|c|c|c|c|c|c|}
\hline \multirow{7}{*}{$\epsilon_{i j} \mapsto$} & \multirow[t]{2}{*}{$I_{j-1}$} & \multicolumn{6}{|c|}{0} & 0 & \multirow{7}{*}{ for $j<i \neq n$, } \\
\hline & & 1 & & $\ldots$ & $\ldots$ & 0 & ${ }^{1}\left(t_{i}-1\right)$ & & \\
\hline & \multirow{4}{*}{0} & 0 & 1 & 0 & $\ldots$ & $\ldots$ & 0 & & \\
\hline & & $\vdots$ & $\vdots$ & & $\ddots$ & & $\vdots$ & 0 & \\
\hline & & 0 & $\begin{array}{l}\cdots \\
\ldots\end{array}$ & $\begin{array}{l}\cdots \\
\ldots\end{array}$ & $\begin{array}{l}1 \\
0\end{array}$ & $\begin{array}{l}0 \\
1\end{array}$ & 0 & & \\
\hline & & 0 & 0 & $\ldots$ & $\ldots$ & 0 & $t_{j}^{-1}$ & & \\
\hline & 0 & & & & 0 & & & $\overline{I_{n-i}}$ & \\
\hline
\end{tabular}

$$
\begin{aligned}
& \epsilon_{i n} \mapsto\left(\begin{array}{c|c|c}
I_{i-1} & 0 & 0 \\
\hline 0 & t_{n}^{-1} & 0 \\
\hline 0 & 0 & I_{n-i-1}
\end{array}\right),
\end{aligned}
$$




$$
\epsilon_{n j} \mapsto\left(\begin{array}{ccc|ccccc}
\multicolumn{3}{c|}{I_{j-1}} & \multicolumn{5}{c}{0} \\
\hline q_{1} & \ldots & q_{j-1} & t_{j}^{-1} & q_{j+1} & \ldots & \ldots & q_{n-1} \\
0 & 0 & 0 & 0 & 1 & 0 & \ldots & 0 \\
\vdots & \vdots & \vdots & \vdots & & \ddots & & \vdots \\
0 & 0 & 0 & 0 & \ldots & 0 & 1 & 0 \\
0 & 0 & 0 & 0 & \ldots & \ldots & 0 & 1
\end{array}\right)
$$

where $q_{k}=-t_{j}^{-1}\left(t_{k}-1\right)$ for all $1 \leq k \neq j \leq n-1$.

Now, we consider the complex specialization of $\hat{\phi}_{G}$ by letting $t_{i}$ be non-zero complex numbers for all $1 \leq i \leq n$ and $t_{n} \neq 1$.

Theorem 4. Let $0 \neq t_{1}, \ldots, t_{n} \in \mathbb{C}$ and $t_{n} \neq 1$. The representation $\hat{\phi}_{G}\left(t_{1}, \ldots, t_{n}\right)$ : $C b_{n} \mapsto G L_{n-1}(\mathbb{C})$ is irreducible if and only if $t_{i} \neq 1$ for all $1 \leq i<n$.

Proof. For the necessary condition, suppose that there exists $1 \leq s<n$ such that $t_{s}=1$.

Since $s \neq n$, it follows that:

- for $i \neq n$ and $1 \leq j \leq n, \hat{\phi}_{G}\left(\epsilon_{i j}\right)\left(e_{s}\right)=e_{s}$ for $s \neq i$. Also $\hat{\phi}_{G}\left(\epsilon_{i j}\right)\left(e_{s}\right)=$ $t_{j}^{-1} e_{s}$ for $s=i$

- for $i=n$ and $1 \leq j \leq n, \hat{\phi}_{G}\left(\epsilon_{n j}\right)\left(e_{s}\right)=e_{s}$.

So $<e_{s}>$ is an invariant subspace of $\mathbb{C}^{n-1}$ under $\hat{\phi}_{G}$, hence $\hat{\phi}_{G}$ is reducible.

For the sufficient condition, suppose that $t_{i} \neq 1$ for all $1 \leq i<n$.

Let $S$ be a non zero invariant subspace of $\mathbb{C}^{n-1}$ under $\hat{\phi}_{G}$, and let $x=\left(x_{1}, x_{2}, \ldots, x_{n-1}\right)$ be a non zero vector in $S$. Fix $1 \leq r \leq n-1$.

- If $x_{r} \neq 0$, then $\hat{\phi}_{G}\left(\epsilon_{r n}\right)(x)-x=\left(t_{n}^{-1}-1\right) x_{r} e_{r} \in S$. But $\left(t_{n}^{-1}-1\right) x_{r} \neq 0$, so $e_{r} \in S$.

- If $x_{r}=0$, then pick $1 \leq j \neq r \leq n-1$ such that $x_{j} \neq 0$. We have $\hat{\phi}_{G}\left(\epsilon_{r j}\right)(x)-x=-t_{j}^{-1}\left(t_{r}-1\right) x_{j} e_{r} \in S$ with $-t_{j}^{-1}\left(t_{r}-1\right) x_{j} \neq 0$, so $e_{r} \in S$.

Hence $e_{r} \in S$ for any $1 \leq r \leq n-1$, and so $S=\mathbb{C}^{n-1}$. Thus $\hat{\phi}_{G}$ is irreducible.

\section{The IRREDUCIBILITY of THE REPRESENTATIONS $\hat{\rho}_{B}$}

The group $C_{n}$ is a semidirect product $C_{n}=C b_{n} \lambda S_{n}$. We let $t_{1}=t_{2}=\ldots=t_{n}$ in the matrix $\hat{\rho}_{G}\left(\epsilon_{i j}\right)$ in order to get a matrix $\hat{\rho}_{B}\left(\epsilon_{i j}\right)$. To each automorphism in $S_{n}$, we assign the matrix of the corresponding permutation of the elements of the base $W_{n}$. In this way we obtain the representation $\hat{\rho}_{B}: C_{n} \mapsto G L_{n}\left(\mathbb{Z}\left[t^{ \pm 1}\right]\right.$ ) (see $[2])$.

Definition 5. [2] The representation $\hat{\rho}_{B}$ is defined as follows

$$
\hat{\rho}_{B}: C_{n} \mapsto G L_{n}\left(\mathbb{Z}\left[t^{ \pm 1}\right]\right)
$$


ON THE IRREDUCIBILITY OF THE EXTENSIONS OF BURAU AND GASSNER REPRESENTATION\$

$$
\begin{aligned}
& \epsilon_{i j} \mapsto\left(\begin{array}{c|cccccc|c}
I_{i-1} & & & & 0 & & & 0 \\
\hline & t^{-1} & 0 & \ldots & \ldots & \ldots & 1-t^{-1} & \\
& 0 & 1 & 0 & \ldots & \ldots & 0 & \\
& 0 & 0 & 1 & 0 & \ldots & 0 & 0 \\
& \vdots & \vdots & & \ddots & & \vdots & \\
& 0 & \ldots & \ldots & 0 & 1 & 0 & \\
& 0 & 0 & \ldots & \ldots & 0 & 1 & \\
\hline 0 & & & & 0 & & & I_{n-j}
\end{array}\right) \text { for } i<j, \\
& \epsilon_{i j} \mapsto\left(\begin{array}{c|cccccc|c}
I_{j-1} & & & 0 & & & & 0 \\
\hline & 1 & 0 & \ldots & \ldots & 0 & 0 & \\
& 0 & 1 & 0 & \ldots & \ldots & 0 & \\
0 & \vdots & \vdots & & \ddots & & \vdots & 0 \\
& 0 & \ldots & 0 & 1 & 0 & 0 & \\
& 0 & \ldots & \ldots & 0 & 1 & 0 & \\
& 1-t^{-1} & 0 & \ldots & \ldots & 0 & t^{-1} & \\
\hline 0 & & & 0 & & & & I_{n-i}
\end{array}\right) \text { for } j<i,
\end{aligned}
$$

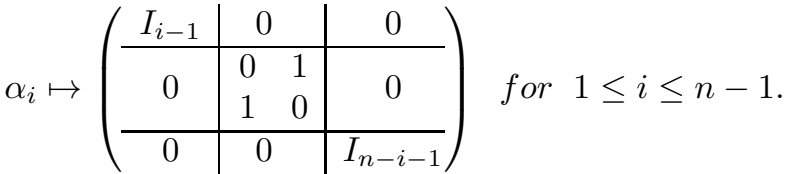

Note that for $\epsilon_{i j}$, in the cases $i=1$ and $j=1$, we omit the first $i-1$ (respectively $j-1$ ) rows and $i-1$ (respectively $j-1$ ) columns. And in the cases $i=n$ and $j=n$, we omit the last $n-i$ (respectively $n-j$ ) rows and $n-i$ (respectively $n-j$ ) columns.

For $\alpha_{i}$ 's, in the case $i=1$, we omit the first $i-1$ rows and $i-1$ columns. And in the case $i=n-1$, we omit the last $n-i-1$ rows and $n-i-1$ columns.

Theorem 6. The representation $\hat{\rho}_{B}$ is reducible.

Proof. Let $v=[1,1, \ldots, 1]^{T}$, where $T$ is the transpose. We see that $\epsilon_{i j}(v)=v$ for all $1 \leq i, j \leq n$, and $\alpha_{i}(v)=v$ for all $1 \leq i \leq n-1$. So $v$ is fixed under the generators of $\hat{\rho}_{B}$. Thus $\hat{\rho}_{B}$ is reducible.

We now specialize $t$ to a non-zero complex number and we find a composition factor of degree $n-1$ of $\hat{\rho}_{B}$. For $\mathbb{C}^{n}$, consider the basis $S=\left\{e_{1}, e_{2}, \ldots, e_{n-1}, v\right\}$, where $v=[1,1, \ldots, 1]^{T}$.

Consider first the action of $\epsilon_{i j}$ 's on the basis $S$.

For $i<j \neq n$ :

$\epsilon_{i j}\left(e_{1}\right)=e_{1}, \epsilon_{i j}\left(e_{2}\right)=e_{2}, \ldots, \epsilon_{i j}\left(e_{i-1}\right)=e_{i-1}, \epsilon_{i j}\left(e_{i}\right)=t^{-1} e_{i}, \epsilon_{i j}\left(e_{i+1}\right)=e_{i+1}$, $\ldots, \epsilon_{i j}\left(e_{j-1}\right)=e_{j-1}, \epsilon_{i j}\left(e_{j}\right)=\left(1-t^{-1}\right) e_{i}+e_{j}, \epsilon_{i j}\left(e_{j+1}\right)=e_{j+1}, \ldots, \epsilon_{i j}\left(e_{n-1}\right)=$ $e_{n-1}, \epsilon_{i j}(v)=v$.

For $j=n$ :

$\epsilon_{i n}\left(e_{1}\right)=e_{1}, \epsilon_{i n}\left(e_{2}\right)=e_{2}, \ldots, \epsilon_{i n}\left(e_{i-1}\right)=e_{i-1}, \epsilon_{i n}\left(e_{i}\right)=t^{-1} e_{i}, \epsilon_{i n}\left(e_{i+1}\right)=e_{i+1}$, $\ldots, \epsilon_{i n}\left(e_{n-1}\right)=e_{n-1}, \ldots, \epsilon_{i n}(v)=v$.

For $j<i \neq n$ :

$\epsilon_{i j}\left(e_{1}\right)=e_{1}, \epsilon_{i j}\left(e_{2}\right)=e_{2}, \ldots, \epsilon_{i j}\left(e_{j-1}\right)=e_{j-1}, \epsilon_{i j}\left(e_{j}\right)=e_{j}+\left(1-t^{-1}\right) e_{i}$, 
$\epsilon_{i j}\left(e_{j+1}\right)=e_{j+1}, \ldots, \epsilon_{i j}\left(e_{i-1}\right)=e_{i-1}, \epsilon_{i j}\left(e_{i}\right)=t^{-1} e_{i}, \epsilon_{i j}\left(e_{i+1}\right)=e_{i+1}, \ldots$, $\epsilon_{i j}\left(e_{n-1}\right)=e_{n-1}, \epsilon_{i j}(v)=v$.

For $i=n$ :

$\epsilon_{n j}\left(e_{1}\right)=e_{1}, \epsilon_{n j}\left(e_{2}\right)=e_{2}, \ldots, \epsilon_{n j}\left(e_{j-1}\right)=e_{j-1}, \epsilon_{n j}\left(e_{j}\right)=\left(t^{-1}-1\right) e_{1}+\left(t^{-1}-\right.$ 1) $e_{2}+\ldots+\left(t^{-1}-1\right) e_{j-1}+t^{-1} e_{j}+\left(t^{-1}-1\right) e_{j+1}+\ldots+\left(t^{-1}-1\right) e_{n-1}+t^{-1} v$, $\epsilon_{n j}\left(e_{j+1}\right)=e_{j+1}, \ldots, \epsilon_{n j}\left(e_{n-1}\right)=e_{n-1}, \epsilon_{n j}(v)=v$.

Now, we consider the action of $\alpha_{i}$ 's on the basis $S$.

For $i \neq n-1$ :

$\alpha_{i}\left(e_{1}\right)=e_{1}, \alpha_{i}\left(e_{2}\right)=e_{2}, \ldots, \alpha_{i}\left(e_{i-1}\right)=e_{i-1}, \alpha_{i}\left(e_{i}\right)=e_{i+1}, \alpha_{i}\left(e_{i+1}\right)=e_{i}$, $\alpha_{i}\left(e_{i+2}\right)=e_{i+2}, \ldots, \alpha_{i}\left(e_{n-1}\right)=e_{n-1}, \alpha_{i}(v)=v$.

For $i=n-1$ :

$\alpha_{n-1}\left(e_{1}\right)=e_{1}, \alpha_{n-1}\left(e_{2}\right)=e_{2}, \ldots, \alpha_{n-1}\left(e_{n-2}\right)=e_{n-2}, \alpha_{n-1}\left(e_{n-1}\right)=-e_{1}-e_{2}-$ $\ldots-e_{n-1}+v, \alpha_{n-1}(v)=v$.

So, the representation $\hat{\rho}_{B}$ in the new basis $S$ becomes as follows

$$
\begin{aligned}
& \epsilon_{i j} \mapsto\left(\begin{array}{c|cccccc|c}
I_{i-1} & & & 0 & & & 0 \\
\hline & t^{-1} & 0 & \ldots & \ldots & \ldots & 0 & \\
& 0 & 1 & 0 & \ldots & \ldots & 0 & \\
0 & 0 & 0 & 1 & 0 & \ldots & 0 & 0 \\
& \vdots & \vdots & & \ddots & & \vdots & 0 \\
& 0 & \ldots & \ldots & 0 & 1 & 0 & \\
& 1-t^{-1} & 0 & \ldots & \ldots & 0 & 1 & \\
\hline 0 & & & 0 & & & & I_{n-j}
\end{array}\right) \text { for } i<j \neq n \\
& \epsilon_{i n} \mapsto\left(\begin{array}{c|c|c}
I_{i-1} & 0 & 0 \\
\hline 0 & t^{-1} & 0 \\
\hline 0 & 0 & I_{n-i}
\end{array}\right),
\end{aligned}
$$

\begin{tabular}{|c|c|c|c|c|c|c|c|c|c|}
\hline \multirow{8}{*}{$\epsilon_{i j} \mapsto$} & \multirow{2}{*}{$I_{j-1}$} & \multicolumn{6}{|c|}{0} & \multirow[t]{2}{*}{0} & \multirow{7}{*}{ for $j<i \neq n$, } \\
\hline & & 1 & 0 & $\cdots$ & $\ldots$ & 0 & $1-t^{-1}$ & & \\
\hline & \multirow{5}{*}{0} & 0 & 1 & 0 & $\ldots$ & ... & 0 & & \\
\hline & & $\vdots$ & $:$ & & $\ddots$ & & $\vdots$ & 0 & \\
\hline & & 0 & . & $\cdots$ & 1 & 0 & 0 & & \\
\hline & & 0 & $\cdots$ & $\cdots$ & 0 & 1 & 0 & & \\
\hline & & 0 & 0 & $\ldots$ & $\cdots$ & 0 & $t^{-1}$ & & \\
\hline & 0 & & & & 0 & & & & \\
\hline
\end{tabular}

$$
\epsilon_{n j} \mapsto\left(\begin{array}{ccc|ccccc}
\multicolumn{3}{c|}{I_{j-1}} & \multicolumn{6}{|c}{0} \\
\hline t^{-1}-1 & \ldots & t^{-1}-1 & t^{-1} & t^{-1}-1 & \ldots & t^{-1}-1 & t^{-1} \\
0 & 0 & 0 & 0 & 1 & 0 & \ldots & 0 \\
\vdots & \vdots & \vdots & \vdots & & \ddots & & \vdots \\
0 & 0 & 0 & 0 & \ldots & 0 & 1 & 0 \\
0 & 0 & 0 & 0 & \ldots & \ldots & 0 & 1
\end{array}\right),
$$


ON THE IRREDUCIBILITY OF THE EXTENSIONS OF BURAU AND GASSNER REPRESENTATIONG

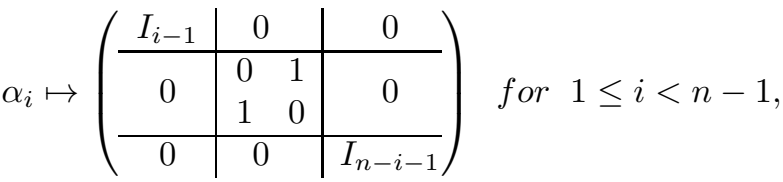

$$
\begin{aligned}
& \alpha_{n-1} \mapsto\left(\begin{array}{cccc|cc}
\multicolumn{4}{c|}{I_{n-2}} & 0 \\
\hline-1 & -1 & \ldots & -1 & -1 & 1 \\
0 & 0 & 0 & 0 & 0 & 1
\end{array}\right) .
\end{aligned}
$$

Now, we remove the last row and the last column to obtain the $n-1$ composition factor $\hat{\phi}_{B}$, which is given by

$$
\begin{aligned}
& \epsilon_{i j} \mapsto\left(\begin{array}{c|cccccc|c}
I_{i-1} & & & 0 & & & 0 \\
\hline & t^{-1} & 0 & \ldots & \ldots & \ldots & 0 & \\
& 0 & 1 & 0 & \ldots & \ldots & 0 & \\
& 0 & 0 & 1 & 0 & \ldots & 0 & 0 \\
& \vdots & \vdots & & \ddots & & \vdots & 0 \\
& 0 & \ldots & \ldots & \ldots & 1 & 0 & \\
& 1-t^{-1} & 0 & \ldots & \ldots & 0 & 1 & \\
\hline 0 & & & 0 & & & & \\
& & & & & &
\end{array}\right. \\
& \epsilon_{i n} \mapsto\left(\begin{array}{c|c|c}
I_{i-1} & 0 & 0 \\
\hline 0 & t^{-1} & 0 \\
\hline 0 & 0 & I_{n-i-1}
\end{array}\right) \text {, }
\end{aligned}
$$$$
\epsilon_{i j} \mapsto\left(\begin{array}{c|cccccc|c}
I_{j-1} & & & & 0 & & & 0 \\
\hline & 1 & 0 & \ldots & \ldots & 0 & 1-t^{-1} & \\
& 0 & 1 & 0 & \ldots & \ldots & 0 & \\
& \vdots & \vdots & & \ddots & & \vdots & 0 \\
& 0 & \ldots & \ldots & 1 & 0 & 0 & \\
& 0 & \ldots & \ldots & 0 & 1 & 0 & \\
& 0 & 0 & \ldots & \ldots & 0 & t^{-1} & \\
\hline 0 & & & & 0 & & & I_{n-i-1}
\end{array}\right) \text { for } j<i \neq n
$$

$$
\begin{aligned}
& \epsilon_{n j} \mapsto\left(\begin{array}{ccc|ccccc}
\multicolumn{3}{c|}{I_{j-1}} & \multicolumn{5}{|c}{0} \\
\hline t^{-1}-1 & \ldots & t^{-1}-1 & t^{-1} & t^{-1}-1 & \ldots & \ldots & t^{-1}-1 \\
0 & 0 & 0 & 0 & 1 & 0 & \ldots & 0 \\
\vdots & \vdots & \vdots & \vdots & & \ddots & & \vdots \\
0 & 0 & 0 & 0 & \ldots & 0 & 1 & 0 \\
0 & 0 & 0 & 0 & \ldots & \ldots & 0 & 1
\end{array}\right),
\end{aligned}
$$

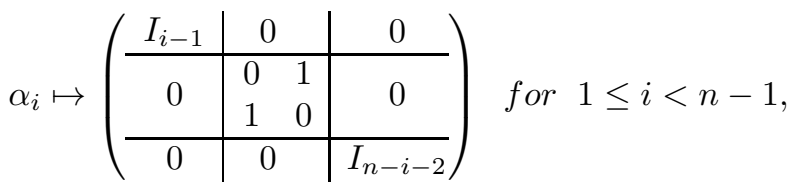

$$
\begin{aligned}
& \left.\alpha_{n-1} \mapsto \begin{array}{llll|l}
\multicolumn{4}{|l|}{I_{n-2}} & 0 \\
\hline-1 & -1 & \ldots & -1 & -1
\end{array}\right) .
\end{aligned}
$$


Now, we consider the complex specialization of $\hat{\phi}_{B}$ by letting $t$ be a non-zero complex number.

Theorem 7. Let $0 \neq t \in \mathbb{C}$. The representation $\hat{\phi}_{B}(t): C_{n} \mapsto G L_{n-1}(\mathbb{C})$ is irreducible.

Proof. Since the restriction of the representation $\hat{\phi}_{B}(t)$ to the subgroup $S_{n}$ inside $C_{n}$ is irreducible, it follows that $\hat{\phi}_{B}(t)$ itself is irreducible.

5. The tensor Product of COMPlex IRREDUCiBle RePresentations of $C b_{3}$

In this section, we set $n=3$ and we consider the complex irreducible specialization $\hat{\phi}_{G}$, which is given by

$$
\begin{gathered}
\epsilon_{12} \mapsto\left(\begin{array}{cc}
t_{2}^{-1} & 0 \\
t_{2}^{-1}\left(t_{1}-1\right) & 1
\end{array}\right), \epsilon_{21} \mapsto\left(\begin{array}{cc}
1 & t_{1}^{-1}\left(t_{2}-1\right) \\
0 & t_{1}^{-1}
\end{array}\right), \epsilon_{13} \mapsto\left(\begin{array}{cc}
t_{3}^{-1} & 0 \\
0 & 1
\end{array}\right), \\
\epsilon_{31} \mapsto\left(\begin{array}{cc}
t_{1}^{-1} & -t_{1}^{-1}\left(t_{2}-1\right) \\
0 & 1
\end{array}\right), \epsilon_{32} \mapsto\left(\begin{array}{cc}
1 & \\
-t_{2}^{-1}\left(t_{1}-1\right) & t_{2}^{-1}
\end{array}\right), \epsilon_{23} \mapsto\left(\begin{array}{cc}
1 & 0 \\
0 & t_{3}^{-1}
\end{array}\right) .
\end{gathered}
$$

Now, we consider the generators of $\hat{\phi}_{G}\left(t_{1}, t_{2}, t_{3}\right) \otimes \hat{\phi}_{G}\left(m_{1}, m_{2}, m_{3}\right)$. For simplicity, we write $\left(\hat{\phi}_{G}\left(t_{1}, t_{2}, t_{3}\right) \otimes \hat{\phi}_{G}\left(m_{1}, m_{2}, m_{3}\right)\right)\left(\epsilon_{i j}\right)=\epsilon_{i j}$.

$$
\begin{aligned}
& \epsilon_{12} \mapsto\left(\begin{array}{cccc}
t_{2}^{-1} m_{2}^{-1} & 0 & 0 & 0 \\
t_{2}^{-1}\left(t_{1}-1\right) m_{2}^{-1} & m_{2}^{-1} & 0 & 0 \\
t_{2}^{-1} m_{2}^{-1}\left(m_{1}-1\right) & 0 & t_{2}^{-1} & 0 \\
t_{2}^{-1}\left(t_{1}-1\right) m_{2}^{-1}\left(m_{1}-1\right) & m_{2}^{-1}\left(m_{1}-1\right) & t_{2}^{-1}\left(t_{1}-1\right) & 1
\end{array}\right), \\
& \epsilon_{21} \mapsto\left(\begin{array}{cccc}
1 & t_{1}^{-1}\left(t_{2}-1\right) & m_{1}^{-1}\left(m_{2}-1\right) & t_{1}^{-1}\left(t_{2}-1\right) m_{1}^{-1}\left(m_{2}-1\right) \\
0 & t_{1}^{-1} & 0 & t_{1}^{-1} m_{1}^{-1}\left(m_{2}-1\right) \\
0 & 0 & m_{1}^{-1} & t_{1}^{-1} m_{1}^{-1}\left(t_{2}-1\right) \\
0 & 0 & 0 & t_{1}^{-1} m_{1}^{-1}
\end{array}\right), \\
& \epsilon_{13} \mapsto\left(\begin{array}{cccc}
t_{3}^{-1} m_{3}^{-1} & 0 & 0 & 0 \\
0 & m_{3}^{-1} & 0 & 0 \\
0 & 0 & t_{3}^{-1} & 0 \\
0 & 0 & 0 & 1
\end{array}\right) \\
& \epsilon_{31} \mapsto\left(\begin{array}{cccc}
t_{1}^{-1} m_{1}^{-1} & -t_{1}^{-1}\left(t_{2}-1\right) m_{1}^{-1} & -t_{1}^{-1} m_{1}^{-1}\left(m_{2}-1\right) & t_{1}^{-1}\left(t_{2}-1\right) m_{1}^{-1}\left(m_{2}-1\right) \\
0 & m_{1}^{-1} & 0 & -m_{1}^{-1}\left(m_{2}-1\right) \\
0 & 0 & t_{1}^{-1} & -t_{1}^{-1}\left(t_{2}-1\right) \\
0 & 0 & 0 & 1
\end{array}\right) \\
& \epsilon_{23} \mapsto\left(\begin{array}{cccc}
1 & 0 & 0 & 0 \\
0 & t_{3}^{-1} & 0 & 0 \\
0 & 0 & m_{3}^{-1} & 0 \\
0 & 0 & 0 & t_{3}^{-1} m_{3}^{-1}
\end{array}\right),
\end{aligned}
$$


ON THE IRREDUCIBILITY OF THE EXTENSIONS OF BURAU AND GASSNER REPRESENTATION\$

$\epsilon_{32} \mapsto\left(\begin{array}{cccc}1 & 0 & 0 & 0 \\ -t_{2}^{-1}\left(t_{1}-1\right) & t_{2}^{-1} & 0 & 0 \\ -m_{2}^{-1}\left(m_{1}-1\right) & 0 & m_{2}^{-1} & 0 \\ t_{2}^{-1}\left(t_{1}-1\right) m_{2}^{-1}\left(m_{1}-1\right) & -t_{2}^{-1} m_{2}^{-1}\left(m_{1}-1\right) & -t_{2}^{-1}\left(t_{1}-1\right) m_{2}^{-1} & t_{2}^{-1} m_{2}^{-1}\end{array}\right)$.

By Theorem 4, we assume that $t_{i} \neq 1$ for all $1 \leq i \leq 3$ and $m_{i} \neq 1$ for all $1 \leq i \leq 3$.

Theorem 8. For $n=3$, the tensor product representation $\hat{\phi}_{G}\left(t_{1}, t_{2}, t_{3}\right) \otimes \hat{\phi}_{G}\left(m_{1}, m_{2}, m_{3}\right)$ is irreducible if and only if $\left(t_{1}, t_{2}, t_{3}\right)$ and $\left(m_{1}, m_{2}, m_{3}\right)$ are distinct vectors.

Proof. For the necessary condition, suppose that $\left(t_{1}, t_{2}, t_{3}\right)$ and $\left(m_{1}, m_{2}, m_{3}\right)$ are equal vectors. Consider $S_{1}=<e_{1}, e_{2}+e_{3}, e_{4}>$.

$$
\begin{aligned}
& \epsilon_{12}\left(e_{1}\right)=t_{2}^{-2} e_{1}+t_{2}^{-2}\left(t_{1}-1\right)\left(e_{2}+e_{3}\right)+t_{2}^{-2}\left(t_{1}-1\right)^{2} e_{4} \in S_{1} \\
& \epsilon_{21}\left(e_{1}\right)=e_{1} \in S_{1} \\
& \epsilon_{13}\left(e_{1}\right)=t_{3}^{-2} e_{1} \in S_{1} \\
& \epsilon_{31}\left(e_{1}\right)=t_{1}^{-2} e_{1} \in S_{1} \\
& \epsilon_{23}\left(e_{1}\right)=e_{1} \in S_{1} \\
& \epsilon_{32}\left(e_{1}\right)=e_{1}-t_{2}^{-1}\left(t_{1}-1\right)\left(e_{2}+e_{3}\right)+\left(t_{1}-1\right)^{2} t_{2}^{-2} e_{4} \in S_{1} \\
& \epsilon_{12}\left(e_{2}+e_{3}\right)=t_{2}^{-1}\left(e_{2}+e_{3}\right)+2 t_{2}^{-1}\left(t_{1}-1\right) e_{4} \in S_{1} \\
& \epsilon_{21}\left(e_{2}+e_{3}\right)=2 t_{1}^{-1}\left(t_{2}-1\right) e_{1}+t_{1}^{-1}\left(e_{2}+e_{3}\right) \in S_{1} \\
& \epsilon_{13}\left(e_{2}+e_{3}\right)=t_{3}^{-1}\left(e_{2}+e_{3}\right) \in S_{1} \\
& \epsilon_{31}\left(e_{2}+e_{3}\right)=-2 t_{1}^{-1}\left(t_{2}-1\right) e_{1}+t_{1}^{-1}\left(e_{2}+e_{3}\right) \in S_{1} \\
& \epsilon_{23}\left(e_{2}+e_{3}\right)=t_{3}^{-1}\left(e_{2}+e_{3}\right) \in S_{1} \\
& \epsilon_{32}\left(e_{2}+e_{3}\right)=t_{2}^{-1}\left(e_{2}+e_{3}\right)-t_{2}^{-2}\left(t_{1}-1\right)^{2} e_{4} \in S_{1} \\
& \epsilon_{12}\left(e_{4}\right)=e_{4} \in S_{1} \\
& \epsilon_{21}\left(e_{4}\right)=t_{1}^{-2}\left(t_{2}-1\right)^{2} e_{1}+t_{1}^{-2}\left(t_{2}-1\right)\left(e_{2}+e_{3}\right)+t_{1}^{-2} e_{4} \in S_{1} \\
& \epsilon_{13}\left(e_{4}\right)=e_{4} \in S_{1} \\
& \epsilon_{31}\left(e_{4}\right)=t_{1}^{-2}\left(t_{2}-1\right)^{2} e_{1}-t_{1}^{-1}\left(t_{2}-1\right)\left(e_{2}+e_{3}\right)+e_{4} \in S_{1} \\
& \epsilon_{23}\left(e_{4}\right)=t_{3}^{-2} e_{4} \in S_{1} \\
& \epsilon_{32}\left(e_{4}\right)=t_{2}^{-2} e_{4} \in S_{1}
\end{aligned}
$$

Therefore, $S_{1}$ is a non trivial invariant subspace of $\mathbb{C}^{4}$ under $\hat{\phi}_{G}\left(t_{1}, t_{2}, t_{3}\right) \otimes \hat{\phi}_{B}\left(m_{1}, m_{2}, m_{3}\right)$. Hence $\hat{\phi}_{G}\left(t_{1}, t_{2}, t_{3}\right) \otimes \hat{\phi}_{B}\left(m_{1}, m_{2}, m_{3}\right)$ is reducible.

For the sufficient condition, suppose that the vectors $\left(t_{1}, t_{2}, t_{3}\right)$ and $\left(m_{1}, m_{2}, m_{3}\right)$ are distinct. Let $S$ be a non trivial invariant subspace of $\mathbb{C}^{4}$ under $\hat{\phi}_{G}\left(t_{1}, t_{2}, t_{3}\right) \otimes$ $\hat{\phi}_{B}\left(m_{1}, m_{2}, m_{3}\right)$.

(a) Suppose $t_{3} \neq m_{3}$ and $t_{3} m_{3} \neq 1$.

In this case, the diagonal matrix $\epsilon_{13}$ has distinct eigenvalues, so $S=\left\langle e_{i}\right\rangle$ or $S=<e_{i}, e_{j}>$ or $S=<e_{i}, e_{j}, e_{k}>$, where $1 \leq i, j, k \leq 4$.

(i) $S \neq<e_{i}>$ for all $1 \leq i \leq 4$.

If $S=<e_{1}>$, then $\epsilon_{12}\left(e_{1}\right)=\beta_{1} e_{1}+\beta_{2} e_{2}+\beta_{3} e_{3}+\beta_{4} e_{4}$ with $\beta_{2}=$ $t_{2}^{-1}\left(t_{1}-1\right) m_{2}^{-1} \neq 0$, which is a contradiction.

If $S=<e_{2}>$, then $\epsilon_{12}\left(e_{2}\right)=\beta_{2} e_{2}+\beta_{4} e_{4}$ with $\beta_{4}=m_{2}^{-1}\left(m_{1}-1\right) \neq 0$, which is a contradiction.

If $S=<e_{3}>$, then $\epsilon_{12}\left(e_{3}\right)=\beta_{3} e_{3}+\beta_{4} e_{4}$ with $\beta_{4}=t_{2}^{-1}\left(t_{1}-1\right) \neq 0$, which is a contradiction. 
If $S=<e_{4}>$, then $\epsilon_{21}\left(e_{4}\right)=\beta_{1} e_{1}+\beta_{2} e_{2}+\beta_{3} e_{3}+\beta_{4} e_{4}$ with $\beta_{1}=$ $t_{1}^{-1}\left(t_{2}-1\right) m_{1}^{-1}\left(m_{2}-1\right) \neq 0$, which is a contradiction.

(ii) $S \neq<e_{i}, e_{j}>$ for all $1 \leq i, j \leq 4$.

If $S=<e_{1}, e_{2}>$, then $\epsilon_{12}\left(e_{1}\right)=\beta_{1} e_{1}+\beta_{2} e_{2}+\beta_{3} e_{3}+\beta_{4} e_{4}$ with $\beta_{3}=t_{2}^{-1} m_{2}^{-1}\left(m_{1}-1\right) \neq 0$, which is a contradiction.

If $S=<e_{1}, e_{3}>$, then $\epsilon_{12}\left(e_{1}\right)=\beta_{1} e_{1}+\beta_{2} e_{2}+\beta_{3} e_{3}+\beta_{4} e_{4}$ with $\beta_{2}=t_{2}^{-1}\left(t_{1}-1\right) m_{2}^{-1} \neq 0$, which is a contradiction.

If $S=<e_{1}, e_{4}>$, then $\epsilon_{12}\left(e_{1}\right)=\beta_{1} e_{1}+\beta_{2} e_{2}+\beta_{3} e_{3}+\beta_{4} e_{4}$ with $\beta_{2}=t_{2}^{-1}\left(t_{1}-1\right) m_{2}^{-1} \neq 0$, which is a contradiction.

If $S=<e_{2}, e_{3}>$, then $\epsilon_{12}\left(e_{2}\right)=\beta_{2} e_{2}+\beta_{4} e_{4}$ with $\beta_{4}=m_{2}^{-1}\left(m_{1}-1\right) \neq$ 0 , which is a contradiction.

If $S=<e_{2}, e_{4}>$, then $\epsilon_{31}\left(e_{2}\right)=\beta_{1} e_{1}+\beta_{2} e_{2}$ with $\beta_{1}=-t_{1}^{-1}\left(t_{2}-\right.$ 1) $m_{1}^{-1} \neq 0$, which is a contradiction.

If $S=<e_{3}, e_{4}>$, then $\epsilon_{31}\left(e_{3}\right)=\beta_{1} e_{1}+\beta_{3} e_{3}$ with $\beta_{1}=-t_{1}^{-1} m_{1}^{-1}\left(m_{2}-\right.$ 1) $\neq 0$, which is a contradiction.

(iii) $S \neq<e_{i}, e_{j}, e_{k}>$ for all $1 \leq i, j, k \leq 4$.

If $S=<e_{1}, e_{2}, e_{3}>$, then $\epsilon_{12}\left(e_{1}\right)=\beta_{1} e_{1}+\beta_{2} e_{2}+\beta_{3} e_{3}+\beta_{4} e_{4}$ with $\beta_{4}=t_{2}^{-1}\left(t_{1}-1\right) m_{2}^{-1}\left(m_{1}-1\right) \neq 0$, which is a contradiction.

If $S=<e_{1}, e_{2}, e_{4}>$, then $\epsilon_{12}\left(e_{1}\right)=\beta_{1} e_{1}+\beta_{2} e_{2}+\beta_{3} e_{3}+\beta_{4} e_{4}$ with $\beta_{3}=t_{2}^{-1} m_{2}^{-1}\left(m_{1}-1\right) \neq 0$, which is a contradiction.

If $S=<e_{1}, e_{3}, e_{4}>$, then $\epsilon_{12}\left(e_{1}\right)=\beta_{1} e_{1}+\beta_{2} e_{2}+\beta_{3} e_{3}+\beta_{4} e_{4}$ with $\beta_{2}=t_{2}^{-1}\left(t_{1}-1\right) m_{2}^{-1} \neq 0$, which is a contradiction.

If $S=<e_{2}, e_{3}, e_{4}>$, then $\epsilon_{31}\left(e_{4}\right)=\beta_{1} e_{1}+\beta_{2} e_{2}+\beta_{3} e_{3}+\beta_{4} e_{4}$ with $\beta_{1}=t_{1}^{-1}\left(t_{2}-1\right) m_{1}^{-1}\left(m_{2}-1\right) \neq 0$, which is a contradiction.

(b) Suppose $t_{3} \neq m_{3}$ and $t_{3} m_{3}=1$.

In addition to the subspaces mentioned in (a), we have other possible candidates to invariant subspaces. More precisely, we consider $S=<$ $a_{1} e_{1}+a_{4} e_{4}>$ or $S=<e_{2}, a_{1} e_{1}+a_{4} e_{4}>$ or $S=<e_{3}, a_{1} e_{1}+a_{4} e_{4}>$ or $S=<e_{2}, e_{3}, a_{1} e_{1}+a_{4} e_{4}>, a_{1} \neq 0$ and $a_{4} \neq 0$.

(i) $S \neq<a_{1} e_{1}+a_{4} e_{4}>$.

If $S=<a_{1} e_{1}+a_{4} e_{4}>$, then $\epsilon_{12}\left(a_{1} e_{1}+a_{4} e_{4}\right)=\beta_{1} e_{1}+\beta_{2} e_{2}+\beta_{3} e_{3}+\beta_{4} e_{4}$ with $\beta_{2}=a_{1} t_{2}^{-1}\left(t_{1}-1\right) m_{2}^{-1} \neq 0$, which is a contradiction.

(ii) $S \neq<e_{2}, a_{1} e_{1}+a_{4} e_{4}>$.

If $S=<e_{2}, a_{1} e_{1}+a_{4} e_{4}>$, then $\epsilon_{12}\left(e_{2}\right)=\beta_{2} e_{2}+\beta_{4} e_{4}$ with $\beta_{4}=$ $m_{2}^{-1}\left(m_{1}-1\right) \neq 0$, and so $e_{4} \in S$. This gives a contradiction.

(iii) $S \neq<e_{3}, a_{1} e_{1}+a_{4} e_{4}>$.

If $S=<e_{3}, a_{1} e_{1}+a_{4} e_{4}>$, then $\epsilon_{12}\left(e_{3}\right)=\beta_{3} e_{3}+\beta_{4} e_{4}$ with $\beta_{4}=$ $t_{2}^{-1}\left(t_{1}-1\right) \neq 0$, and so $e_{4} \in S$. This gives a contradiction.

(iv) $S \neq<e_{2}, e_{3}, a_{1} e_{1}+a_{4} e_{4}>$.

If $S=<e_{2}, e_{3}, a_{1} e_{1}+a_{4} e_{4}>$, then $\epsilon_{12}\left(e_{2}\right)=\beta_{2} e_{2}+\beta_{4} e_{4}$ with $\beta_{4}=$ $m_{2}^{-1}\left(m_{1}-1\right) \neq 0$, and so $e_{4} \in S$. This gives a contradiction.

(c) Suppose $t_{3}=m_{3}$ and $t_{3} m_{3} \neq 1$, then $t_{1} \neq m_{1}$ or $t_{2} \neq m_{2}$.

In addition to the subspaces mentioned in (a), we also have other possible invariant subspaces. For instance, we consider $S=<a_{2} e_{2}+a_{3} e_{3}>$ or $S=<$ $e_{1}, a_{2} e_{2}+a_{3} e_{3}>$ or $S=<e_{4}, a_{2} e_{2}+a_{3} e_{3}>$ or $S=<e_{1}, e_{4}, a_{2} e_{2}+a_{3} e_{3}>$, $a_{2} \neq 0$ and $a_{3} \neq 0$.

(i) $S \neq<a_{2} e_{2}+a_{3} e_{3}>$.

If $S=<a_{2} e_{2}+a_{3} e_{3}>$, then $\epsilon_{12}\left(a_{2} e_{2}+a_{3} e_{3}\right)=m_{2}^{-1} a_{2} e_{2}+t_{2}^{-1} a_{3} e_{3}+$ 
ON THE IRREDUCIBILITY OF THE EXTENSIONS OF BURAU AND GASSNER REPRESENTATIONS

$\gamma_{4} e_{4} \in S$ and $\epsilon_{31}\left(a_{2} e_{2}+a_{3} e_{3}\right)=m_{1}^{-1} a_{2} e_{2}+t_{1}^{-1} a_{3} e_{3}+\delta_{4} e_{4} \in S$, where $\gamma_{4}$ and $\delta_{4}$ are scalars. So $\epsilon_{12}\left(a_{2} e_{2}+a_{3} e_{3}\right)=\lambda_{1}\left(a_{2} e_{2}+a_{3} e_{3}\right)$ and $\epsilon_{31}\left(a_{2} e_{2}+a_{3} e_{3}\right)=\lambda_{2}\left(a_{2} e_{2}+a_{3} e_{3}\right)$. Thus we get $\lambda_{1} a_{2}=m_{2}^{-1} a_{2}$ and $\lambda_{1} a_{3}=t_{2}^{-1} a_{3}$, which implies that $\lambda_{1}=t_{2}^{-1}=m_{2}^{-1}$. Similarly, we have $\lambda_{2} a_{2}=m_{1}^{-1} a_{2}$ and $\lambda_{2} a_{3}=t_{1}^{-1} a_{3}$, which implies that $\lambda_{2}=t_{1}^{-1}=m_{1}^{-1}$. This contradicts the fact that $t_{1} \neq m_{1}$ or $t_{2} \neq m_{2}$.

(ii) $S \neq<e_{1}, a_{2} e_{2}+a_{3} e_{3}>$.

If $S=<e_{1}, a_{2} e_{2}+a_{3} e_{3}>$, then $\epsilon_{12}\left(e_{1}\right)=\beta_{1} e_{1}+\beta_{2} e_{2}+\beta_{3} e_{3}+\beta_{4} e_{4}$ with $\beta_{4}=t_{2}^{-1}\left(t_{1}-1\right) m_{2}^{-1}\left(m_{1}-1\right) \neq 0$, which is a contradiction.

(iii) $S \neq<e_{4}, a_{2} e_{2}+a_{3} e_{3}>$.

If $S=<e_{4}, a_{2} e_{2}+a_{3} e_{3}>$, then $\epsilon_{21}\left(e_{4}\right)=\beta_{1} e_{1}+\beta_{2} e_{2}+\beta_{3} e_{3}+\beta_{4} e_{4}$ with $\beta_{1}=t_{1}^{-1}\left(t_{2}-1\right) m_{1}^{-1}\left(m_{2}-1\right) \neq 0$, which is a contradiction.

(iv) $S \neq<e_{1}, e_{4}, a_{2} e_{2}+a_{3} e_{3}>$.

If $S=<e_{1}, e_{4}, a_{2} e_{2}+a_{3} e_{3}>$, then $\epsilon_{12}\left(a_{2} e_{2}+a_{3} e_{3}\right)=m_{2}^{-1} a_{2} e_{2}+$ $t_{2}^{-1} a_{3} e_{3}+\gamma_{4} e_{4} \in S$ and $\epsilon_{31}\left(a_{2} e_{2}+a_{3} e_{3}\right)=m_{1}^{-1} a_{2} e_{2}+t_{1}^{-1} a_{3} e_{3}+\delta_{4} e_{4} \in$ $S$, where $\gamma_{4}$ and $\delta_{4}$ are scalars. So $\epsilon_{12}\left(a_{2} e_{2}+a_{3} e_{3}\right)=\lambda_{1}\left(a_{2} e_{2}+a_{3} e_{3}\right)+$ $\gamma_{1} e_{1}+\omega_{1} e_{4}$ and $\epsilon_{31}\left(a_{2} e_{2}+a_{3} e_{3}\right)=\lambda_{2}\left(a_{2} e_{2}+a_{3} e_{3}\right)+\gamma_{2} e_{1}+\omega_{2} e_{4}$, where $\lambda_{1}, \lambda_{2}, \gamma_{1}, \gamma_{2}, \omega_{1}$, and $\omega_{2}$ are non-zero scalars. Thus we get $\lambda_{1} a_{2}=m_{2}^{-1} a_{2}$ and $\lambda_{1} a_{3}=t_{2}^{-1} a_{3}$, which implies that $\lambda_{1}=t_{2}^{-1}=m_{2}^{-1}$. Similarly, we have $\lambda_{2} a_{2}=m_{1}^{-1} a_{2}$ and $\lambda_{2} a_{3}=t_{1}^{-1} a_{3}$, which implies that $\lambda_{2}=t_{1}^{-1}=m_{1}^{-1}$. This contradicts the fact that $t_{1} \neq m_{1}$ or $t_{2} \neq m_{2}$.

(d) Suppose $t_{3}=m_{3}$ and $t_{3} m_{3}=1$, then $t_{1} \neq m_{1}$ or $t_{2} \neq m_{2}$.

In addition to all previous subspaces mentioned in (a), (b) and (c), we may also consider $S=<a_{1} e_{1}+a_{4} e_{4}, a_{2} e_{2}+a_{3} e_{3}>$ with $a_{i} \neq 0$ for all $1 \leq i \leq 4$.

If $S=<a_{1} e_{1}+a_{4} e_{4}, a_{2} e_{2}+a_{3} e_{3}>$, then $\epsilon_{12}\left(a_{2} e_{2}+a_{3} e_{3}\right)=m_{2}^{-1} a_{2} e_{2}+$ $t_{2}^{-1} a_{3} e_{3}+\gamma_{4} e_{4} \in S$ and $\epsilon_{31}\left(a_{2} e_{2}+a_{3} e_{3}\right)=m_{1}^{-1} a_{2} e_{2}+t_{1}^{-1} a_{3} e_{3}+$ $\delta_{4} e_{4} \in S$, where $\gamma_{4}$ and $\delta_{4}$ are non-zero scalars. So $\epsilon_{12}\left(a_{2} e_{2}+a_{3} e_{3}\right)=$ $\lambda_{1}\left(a_{2} e_{2}+a_{3} e_{3}\right)+\gamma_{1}\left(a_{1} e_{1}+a_{4} e_{4}\right)$ and $\epsilon_{31}\left(a_{2} e_{2}+a_{3} e_{3}\right)=\lambda_{2}\left(a_{2} e_{2}+\right.$ $\left.a_{3} e_{3}\right)+\gamma_{2}\left(a_{1} e_{1}+a_{4} e_{4}\right)$, where $\lambda_{1}, \lambda_{2}, \gamma_{1}$, and $\gamma_{2}$ are non-zero scalars. So $\lambda_{1} a_{2}=m_{2}^{-1} a_{2}$ and $\lambda_{1} a_{3}=t_{2}^{-1} a_{3}$, which implies that $\lambda_{1}=t_{2}^{-1}=$ $m_{2}^{-1}$. Similarly, $\lambda_{2} a_{2}=m_{1}^{-1} a_{2}$ and $\lambda_{2} a_{3}=t_{1}^{-1} a_{3}$, which implies that $\lambda_{2}=t_{1}^{-1}=m_{1}^{-1}$. This contradicts the fact that $t_{1} \neq m_{1}$ or $t_{2} \neq m_{2}$.

Thus, $\mathbb{C}^{4}$ has no non trivial invariant subspace under $\hat{\phi}_{G}\left(t_{1}, t_{2}, t_{3}\right) \otimes \hat{\phi}_{B}\left(m_{1}, m_{2}, m_{3}\right)$. Therefore $\hat{\phi}_{G}\left(t_{1}, t_{2}, t_{3}\right) \otimes \hat{\phi}_{B}\left(m_{1}, m_{2}, m_{3}\right)$ is irreducible.

\section{The tensor PRoduct of COMPlex IRREDUCIBle REPRESEntations of $C_{3}$}

In this section, we set $n=3$ and we consider the irreducible complex specialization $\hat{\phi}_{B}$, which is given by

$$
\begin{gathered}
\epsilon_{12} \mapsto\left(\begin{array}{cc}
t^{-1} & 0 \\
1-t^{-1} & 1
\end{array}\right), \epsilon_{21} \mapsto\left(\begin{array}{cc}
1 & 1-t^{-1} \\
0 & t^{-1}
\end{array}\right), \epsilon_{13} \mapsto\left(\begin{array}{cc}
t^{-1} & 0 \\
0 & 1
\end{array}\right), \\
\epsilon_{31} \mapsto\left(\begin{array}{cc}
t^{-1} & t^{-1}-1 \\
0 & 1
\end{array}\right), \epsilon_{32} \mapsto\left(\begin{array}{cc}
1 & 0 \\
t^{-1}-1 & t^{-1}
\end{array}\right), \epsilon_{23} \mapsto\left(\begin{array}{cc}
1 & 0 \\
0 & t^{-1}
\end{array}\right),
\end{gathered}
$$




$$
\alpha_{1} \mapsto\left(\begin{array}{cc}
0 & 1 \\
1 & 0
\end{array}\right), \alpha_{2} \mapsto\left(\begin{array}{cc}
1 & 0 \\
-1 & -1
\end{array}\right) .
$$

Now, we consider the generators of $\hat{\phi}_{B}(t) \otimes \hat{\phi}_{B}(m)$. For simplicity, we set $\left(\hat{\phi}_{B}(t) \otimes\right.$ $\left.\hat{\phi}_{B}(m)\right)\left(\epsilon_{i j}\right)=\epsilon_{i j}$ and $\left(\hat{\phi}_{B}(t) \otimes \hat{\phi}_{B}(m)\right)\left(\alpha_{i}\right)=\alpha_{i}$.

$$
\begin{aligned}
& \epsilon_{12} \mapsto\left(\begin{array}{cccc}
t^{-1} m^{-1} & 0 & 0 & 0 \\
t^{-1}\left(1-m^{-1}\right) & t^{-1} & 0 & 0 \\
m^{-1}\left(1-t^{-1}\right) & 0 & m^{-1} & 0 \\
\left(1-t^{-1}\right)\left(1-m^{-1}\right) & \left(1-t^{-1}\right) & \left(1-m^{-1}\right) & 1
\end{array}\right), \\
& \epsilon_{21} \mapsto\left(\begin{array}{cccc}
1 & 1-m^{-1} & 1-t^{-1} & \left(1-t^{-1}\right)\left(1-m^{-1}\right) \\
0 & m^{-1} & 0 & m^{-1}\left(1-t^{-1}\right) \\
0 & 0 & t^{-1} & t^{-1}\left(1-m^{-1}\right) \\
0 & 0 & 0 & t^{-1} m^{-1}
\end{array}\right) \\
& \epsilon_{13} \mapsto\left(\begin{array}{cccc}
t^{-1} m^{-1} & 0 & 0 & 0 \\
0 & t^{-1} & 0 & 0 \\
0 & 0 & m^{-1} & 0 \\
0 & 0 & 0 & 1
\end{array}\right) \\
& \epsilon_{31} \mapsto\left(\begin{array}{cccc}
t^{-1} m^{-1} & t^{-1}\left(m^{-1}-1\right) & m^{-1}\left(t^{-1}-1\right) & \left(t^{-1}-1\right)\left(m^{-1}-1\right) \\
0 & t^{-1} & 0 & t^{-1}-1 \\
0 & 0 & m^{-1} & m^{-1}-1 \\
0 & 0 & 0 & 1
\end{array}\right) \\
& \epsilon_{23} \mapsto\left(\begin{array}{cccc}
1 & 0 & 0 & 0 \\
0 & m^{-1} & 0 & 0 \\
0 & 0 & t^{-1} & 0 \\
0 & 0 & 0 & t^{-1} m^{-1}
\end{array}\right) \\
& \epsilon_{32} \mapsto\left(\begin{array}{cccc}
1 & 0 & 0 & 0 \\
m^{-1}-1 & m^{-1} & 0 & 0 \\
t^{-1}-1 & 0 & t^{-1} & 0 \\
\left(t^{-1}-1\right)\left(m^{-1}-1\right) & m^{-1}\left(t^{-1}-1\right) & t^{-1}\left(m^{-1}-1\right) & t^{-1} m^{-1}
\end{array}\right), \\
& \alpha_{1} \mapsto\left(\begin{array}{cccc}
0 & 0 & 0 & 1 \\
0 & 0 & 1 & 0 \\
0 & 1 & 0 & 0 \\
1 & 0 & 0 & 0
\end{array}\right) \\
& \alpha_{2} \mapsto\left(\begin{array}{cccc}
1 & 0 & 0 & 0 \\
-1 & -1 & 0 & 0 \\
-1 & 0 & -1 & 0 \\
1 & 1 & 1 & 1
\end{array}\right) .
\end{aligned}
$$

Theorem 9. For $n=3$, the tensor product representation $\hat{\phi}_{B}(t) \otimes \hat{\phi}_{B}(m)$ is irreducible if and only if $t \neq m$.

Proof. For the necessary condition, we suppose that $t=m$, and we consider $S_{1}=$ $\left\{e_{1}, e_{2}+e_{3}, e_{4}\right\}$.

$\epsilon_{12}\left(e_{1}\right)=t^{-2} e_{1}+t^{-1}\left(1-t^{-1}\right)\left(e_{2}+e_{3}\right)+\left(1-t^{-1}\right)^{2} e_{4} \in S_{1}$

$\epsilon_{21}\left(e_{1}\right)=e_{1} \in S_{1}$ 


$$
\begin{aligned}
& \epsilon_{13}\left(e_{1}\right)=e_{1} \in S_{1} \\
& \epsilon_{31}\left(e_{1}\right)=t^{-2} e_{1} \in S_{1} \\
& \epsilon_{23}\left(e_{1}\right)=e_{1} \in S_{1} \\
& \epsilon_{32}\left(e_{1}\right)=e_{1}+\left(t^{-1}-1\right)\left(e_{2}+e_{3}\right)+\left(t^{-1}-1\right)^{2} e_{4} \in S_{1} \\
& \epsilon_{12}\left(e_{2}+e_{3}\right)=t^{-1}\left(e_{2}+e_{3}\right)+2\left(1-t^{-1}\right) e_{4} \in S_{1} \\
& \epsilon_{21}\left(e_{2}+e_{3}\right)=2\left(1-t^{-1}\right) e_{1}+t^{-1}\left(e_{2}+e_{3}\right) \in S_{1} \\
& \epsilon_{13}\left(e_{2}+e_{3}\right)=t^{-1}\left(e_{2}+e_{3}\right) \in S_{1} \\
& \epsilon_{31}\left(e_{2}+e_{3}\right)=2 t^{-1}\left(1-t^{-1}\right) e_{1}+t^{-1}\left(e_{2}+e_{3}\right) \in S_{1} \\
& \epsilon_{23}\left(e_{2}+e_{3}\right)=t^{-1}\left(e_{2}+e_{3}\right) \in S_{1} \\
& \epsilon_{32}\left(e_{2}+e_{3}\right)=t^{-1}\left(e_{2}+e_{3}\right)+t^{-1}\left(t^{-1}-1\right) e_{4} \in S_{1} \\
& \epsilon_{12}\left(e_{4}\right)=e_{4} \in S_{1} \\
& \epsilon_{21}\left(e_{4}\right)=\left(1-t^{-1}\right)^{2} e_{1}+t^{-1}\left(1-t^{-1}\right)\left(e_{2}+e_{3}\right)+t^{-2} e_{4} \in S_{1} \\
& \epsilon_{13}\left(e_{4}\right)=t^{-2} e_{4} \in S_{1} \\
& \epsilon_{31}\left(e_{4}\right)=\left(t^{-1}-1\right)^{2} e_{1}+\left(t^{-1}-1\right)\left(e_{2}+e_{3}\right)+e_{4} \in S_{1} \\
& \epsilon_{23}\left(e_{4}\right)=t^{-2} e_{4} \in S_{1} \\
& \epsilon_{32}\left(e_{4}\right)=t^{-2} e_{4} \in S_{1}
\end{aligned}
$$

Therefore, $S_{1}$ is a non trivial invariant subspace of $\mathbb{C}^{4}$ under $\hat{\phi}_{B}(t) \otimes \hat{\phi}_{B}(m)$, and so $\hat{\phi}_{B}(t) \otimes \hat{\phi}_{B}(m)$ is reducible.

For the sufficient condition, we suppose $t \neq m, t m \neq 1, t \neq 1$, and $m \neq 1$, then we get that $\epsilon_{23}$ has distinct eigenvalues. Suppose $S$ is a non trivial invariant subspace of $\mathbb{C}^{4}$ under $\hat{\phi}_{B}(t) \otimes \hat{\phi}_{B}(m)$, then $S=<e_{i}>$ or $S=<e_{i}, e_{j}>$ or $S=<e_{i}, e_{j}, e_{k}>$, where $1 \leq i, j, k \leq 4$.

(a) $S \neq<e_{i}>$ for all $1 \leq i \leq 4$.

If $S=<e_{1}>$, then $\alpha_{1}\left(e_{1}\right)=e_{4} \notin S$, which is a contradiction. If $S=<e_{2}>$, then $\alpha_{1}\left(e_{2}\right)=e_{3} \notin S$, which is a contradiction. If $S=<e_{3}>$, then $\alpha_{1}\left(e_{3}\right)=e_{2} \notin S$, which is a contradiction. If $S=<e_{4}>$, then $\alpha_{1}\left(e_{4}\right)=e_{1} \notin S$, which is a contradiction.

(b) $S \neq<e_{i}, e_{j}>$ for all $1 \leq i, j \leq 4$.

If $S=<e_{1}, e_{2}>$, then $\alpha_{1}\left(e_{1}\right)=e_{4} \notin S$, which is a contradiction. If $S=<e_{1}, e_{3}>$, then $\alpha_{1}\left(e_{1}\right)=e_{4} \notin S$, which is a contradiction. If $S=<e_{1}, e_{4}>$, then $\alpha_{2}\left(e_{1}\right)=e_{1}-e_{2}-e_{3}+e_{4} \notin S$, which is a contradiction.

If $S=<e_{2}, e_{3}>$, then $\alpha_{2}\left(e_{2}\right)=-e_{2}+e_{4} \notin S$, which is a contradiction. If $S=<e_{2}, e_{4}>$, then $\alpha_{1}\left(e_{2}\right)=e_{3} \notin S$, which is a contradiction. If $S=<e_{3}, e_{4}>$, then $\alpha_{1}\left(e_{3}\right)=e_{2} \notin S$, which is a contradiction.

(c) $S \neq<e_{i}, e_{j}, e_{k}>$ for all $1 \leq i, j, k \leq 4$.

If $S=<e_{1}, e_{2}, e_{3}>$, then $\alpha_{1}\left(e_{1}\right)=e_{4} \notin S$, which is a contradiction. If $S=<e_{1}, e_{2}, e_{4}>$, then $\alpha_{1}\left(e_{2}\right)=e_{3} \notin S$, which is a contradiction. If $S=<e_{1}, e_{3}, e_{4}>$, then $\alpha_{1}\left(e_{3}\right)=e_{2} \notin S$, which is a contradiction. If $S=<e_{2}, e_{3}, e_{4}>$, then $\alpha_{1}\left(e_{4}\right)=e_{1} \notin S$, which is a contradiction.

Thus $\hat{\phi}_{B}(t) \otimes \hat{\phi}_{B}(m)$ is irreducible in this case.

We then assume that $t \neq m, t m \neq 1$ and $t=1$. It is then clear that $m \neq 1$. Suppose that $S$ is a non trivial invariant subspace of $\mathbb{C}^{4}$ under $\hat{\phi}_{B}(t) \otimes \hat{\phi}_{B}(m)$. In addition to the previous subspaces, we have other possible candidates to invariant subspaces.

(a) If $\operatorname{dim}(S)=1$, then we consider $S=<\beta_{1} e_{1}+\beta_{3} e_{3}>$ or $S=<\beta_{2} e_{2}+\beta_{4} e_{4}>$, where $\beta_{i} \neq 0$ for all $1 \leq i \leq 4$. 
We have $\epsilon_{32}\left(\beta_{1} e_{1}+\beta_{3} e_{3}\right)=\beta_{1} e_{1}+\beta_{1}\left(m^{-1}-1\right) e_{2}+\beta_{3} e_{3}+\beta_{3}\left(m^{-1}-1\right) e_{4}$ with $\beta_{3}\left(m^{-1}-1\right) \neq 0$, which is a contradiction.

Similarly, we have $\epsilon_{21}\left(\beta_{2} e_{2}+\beta_{4} e_{4}\right)=\beta_{2}\left(1-m^{-1}\right) e_{1}+\beta_{2} m^{-1} e_{2}+\beta_{4}(1-$ $\left.m^{-1}\right) e_{3}+\beta_{4} m^{-1} e_{4}$ with $\beta_{2}\left(1-m^{-1}\right) \neq 0$, which is a contradiction.

(b) If $\operatorname{dim}(S)=2$, then we consider $S=<\beta_{1} e_{1}+\beta_{3} e_{3}, \beta_{2} e_{2}+\beta_{4} e_{4}>$. Without loss of generality, we assume that all $\beta_{i}^{\prime} s$ are non zeros. We have $\epsilon_{32}\left(\beta_{1} e_{1}+\right.$ $\left.\beta_{3} e_{3}\right)=\beta_{1} e_{1}+\beta_{1}\left(m^{-1}-1\right) e_{2}+\beta_{3} e_{3}+\beta_{3}\left(m^{-1}-1\right) e_{4}$, and so $\beta_{1} \beta_{4}=$ $\beta_{2} \beta_{3}$. Now, $\alpha_{2}\left(\beta_{1} e_{1}+\beta_{3} e_{3}\right)=\beta_{1} e_{1}-\beta_{1} e_{2}+\left(-\beta_{1}-\beta_{3}\right) e_{3}+\left(\beta_{1}+\beta_{3}\right) e_{4}$, and so $-\beta_{1}-\beta_{3}=\beta_{3}$. Thus we get $\beta_{3}=-\frac{1}{2} \beta_{1}$. On the other hand, $\alpha_{1}\left(\beta_{1} e_{1}+\beta_{3} e_{3}\right)=\beta_{3} e_{2}+\beta_{1} e_{4} \in S$, so $\beta_{3} e_{2}+\beta_{1} e_{4}=k\left(\beta_{2} e_{2}+\beta_{4} e_{4}\right)$ for some non zero constant $k$. Thus we have $\beta_{1} \beta_{2}=\beta_{3} \beta_{4}$, which implies that $\beta_{1} \beta_{2} \beta_{4}=\beta_{3} \beta_{4}^{2}$. Having $\beta_{1} \beta_{4}=\beta_{2} \beta_{3}$, we get $\beta_{2}^{2} \beta_{3}=\beta_{3} \beta_{4}^{2}$ and so $\beta_{2}^{2}=\beta_{4}^{2}$. This means that $\beta_{2}= \pm \beta_{4}$, and so $\beta_{1}= \pm \beta_{3}$. This contradicts the fact that $\beta_{3}=-\frac{1}{2} \beta_{1}$.

(c) If $\operatorname{dim}(S)=3$, then we assume, without loss of generality, that $S=<$ $\beta_{1} e_{1}+\beta_{3} e_{3}, \beta_{2} e_{2}+\beta_{4} e_{4}, e_{1}>$, where $\beta_{i} \neq 0$ for all $1 \leq i \leq 4$. Since we have $e_{1} \in S$ and $\beta_{1} e_{1}+\beta_{3} e_{3} \in S$, it follows that $e_{3} \in S$. On the other hand, $\alpha_{1}\left(e_{1}\right)=e_{4} \in S$ and $\beta_{2} e_{2}+\beta_{4} e_{4} \in S$, which implies that $e_{2} \in S$ and so $S=\mathbb{C}^{4}$. This also gives a contradiction.

Thus $\hat{\phi}_{B}(t) \otimes \hat{\phi}_{B}(m)$ is irreducible in this case.

Now, we suppose that $t \neq m$ and $t m=1$. It follows that $t \neq 1$ and $m \neq 1$. Suppose $S$ is an invariant nontrivial subspace of $\mathbb{C}^{4}$ under $\hat{\phi}_{B}(t) \otimes \hat{\phi}_{B}(m)$.

(a) $e_{i} \notin S$ for any $i=1,2,3,4$.

If $e_{2} \in S$, then $\epsilon_{32}\left(e_{2}\right)=t e_{2}+(1-t) e_{4}=X \in S$. Now, we have $X-t e_{2}=$ $(1-t) e_{4} \in S$, which implies that $e_{4} \in S$. Also, $\epsilon_{31}\left(e_{2}\right)=\left(t^{-1}-1\right) e_{1}+$ $t^{-1} e_{2}=Y \in S$, and so $Y-t^{-1} e_{2}=\left(t^{-1}-1\right) e_{1} \in S$. This implies that $e_{1} \in S$.

So

$$
e_{2} \in S \Longrightarrow e_{1}, e_{4} \in S
$$

Similarly, if $e_{3} \in S$, then $\epsilon_{21}\left(e_{3}\right)=\left(1-t^{-1}\right) e_{1}+t^{-1} e_{3}=X \in S$. Now, we have $X-t^{-1} e_{3}=\left(1-t^{-1}\right) e_{1} \in S$, which implies that $e_{1} \in S$. Also, $\epsilon_{32}\left(e_{3}\right)=t^{-1} e_{3}+\left(1-t^{-1}\right) e_{4}=Y \in S$, and so $Y-t^{-1} e_{3}=\left(1-t^{-1}\right) e_{4} \in S$. This implies that $e_{4} \in S$.

So

$$
e_{3} \in S \Longrightarrow e_{1}, e_{4} \in S
$$

Now, if $e_{1} \in S$, then $\epsilon_{12}\left(e_{1}\right)=e_{1}+\left(t^{-1}-1\right) e_{2}+(t-1) e_{3}+\left(2-t-t^{-1}\right) e_{4}=$ $X \in S$, and $\epsilon_{32}\left(e_{1}\right)=e_{1}+(t-1) e_{2}+\left(t^{-1}-1\right) e_{3}+\left(2-t-t^{-1}\right) e_{4}=Y \in S$. Then $X-Y=\left(-t+t^{-1}\right)\left(e_{2}-e_{3}\right) \in S$, which implies that $e_{2}-e_{3} \in S$. So

$$
e_{1} \in S \Longrightarrow e_{2}-e_{3} \in S
$$

Similarly, if $e_{4} \in S$, then $\epsilon_{21}\left(e_{4}\right)=\left(2-t-t^{-1}\right) e_{1}+(t-1) e_{2}+\left(t^{-1}-1\right) e_{3}+e_{4}=$ $X \in S$, and $\epsilon_{31}\left(e_{4}\right)=\left(2-t-t^{-1}\right) e_{1}+\left(t^{-1}-1\right) e_{2}+(t-1) e_{3}+e_{4}=Y \in S$. Then $X-Y=\left(t-t^{-1}\right)\left(e_{2}-e_{3}\right) \in S$, which implies that $e_{2}-e_{3} \in S$.

So

$$
e_{4} \in S \Longrightarrow e_{2}-e_{3} \in S
$$

Now, suppose that $e_{2} \in S$. Then by (1), we have $e_{1}$ and $e_{4} \in S$, and so by (3), we get $e_{2}-e_{3} \in S$. This implies that $e_{3} \in S$, and so $S=\mathbb{C}^{4}$, which is 
ON THE IRREDUCIBILITY OF THE EXTENSIONS OF BURAU AND GASSNER REPRESENTATION\$

a contradiction. Hence $e_{2} \notin S$.

Similarly, suppose that $e_{3} \in S$. Then by (2), we have $e_{1}$ and $e_{4} \in S$, and so by (3), we get $e_{2}-e_{3} \in S$. This implies that $e_{2} \in S$, and so $S=\mathbb{C}^{4}$, which is a contradiction. Hence $e_{3} \notin S$.

Now, suppose that $e_{1} \in S$. Then $\epsilon_{12}\left(e_{1}\right)-e_{1}=\left(t^{-1}-1\right) e_{2}+(t-1) e_{3}+(2-$ $\left.t-t^{-1}\right) e_{4}=X \in S$ and $\epsilon_{32}\left(e_{1}\right)-e_{1}=(t-1) e_{2}+\left(t^{-1}-1\right) e_{3}+\left(2-t-t^{-1}\right) e_{4}=$ $Y \in S$. So $Z=X+Y=\left(2-t-t^{-1}\right)\left(-e_{2}-e_{3}+2 e_{4}\right) \in S$, which means that $W=-e_{2}-e_{3}+2 e_{4} \in S$. But $\epsilon_{31}(Y)+X=2\left(2-t-t^{-1}\right) e_{4} \in S$, which implies that $e_{4} \in S$, and so $W-2 e_{4}=-e_{2}-e_{3} \in S$. By (3), we have $e_{2}-e_{3} \in S$, so $e_{2} \in S$, which is a contradiction. Hence $e_{1} \notin S$.

Similarly, suppose that $e_{4} \in S$. Then $\epsilon_{21}\left(e_{4}\right)-e_{4}=\left(2-t-t^{-1}\right) e_{1}+(t-1) e_{2}+$ $\left(t^{-1}-1\right) e_{3}=X \in S$ and $\epsilon_{31}\left(e_{4}\right)-e_{4}=\left(2-t-t^{-1}\right) e_{1}+\left(t^{-1}-1\right) e_{2}+(t-1) e_{3}=$ $Y \in S$. So $Z=X+Y=\left(2-t-t^{-1}\right)\left(2 e_{1}-e_{2}-e_{3}\right) \in S$, which means that $W=2 e_{1}-e_{2}-e_{3} \in S$. But $\epsilon_{23}(y)+X=2\left(2-t-t^{-1}\right) e_{1} \in S$, which implies that $e_{1} \in S$, and then $W-2 e_{1}=-e_{2}-e_{3} \in S$. By (4), we have $e_{2}-e_{3} \in S$, so $e_{2} \in S$, which is a contradiction. Hence $e_{4} \notin S$.

Therefore, $e_{i} \notin S$ for any $i=1,2,3,4$.

(b) $\alpha_{i} e_{i}+\alpha_{j} e_{j} \notin S$ for any $1 \leq i \neq j \leq 4$.

Suppose that $\alpha_{1} e_{1}+\alpha_{2} e_{2} \in S$ with $\alpha_{1} \neq 0$ and $\alpha_{2} \neq 0$. Then $\epsilon_{23}\left(\alpha_{1} e_{1}+\right.$ $\left.\alpha_{2} e_{2}\right)=\alpha_{1} e_{1}+t \alpha_{2} e_{2}=X \in S$, and so $X-\left(\alpha_{1} e_{1}+\alpha_{2} e_{2}\right) \in S$. This implies that $-(t-1) \alpha_{2} e_{2} \in S$. And so $e_{2} \in S$, which contradicts (a). Thus $\alpha_{1} e_{1}+\alpha_{2} e_{2} \notin S$.

Suppose that $\alpha_{1} e_{1}+\alpha_{3} e_{3} \in S$ with $\alpha_{1} \neq 0$ and $\alpha_{3} \neq 0$. Then $\epsilon_{23}\left(\alpha_{1} e_{1}+\right.$ $\left.\alpha_{3} e_{3}\right)=\alpha_{1} e_{1}+t^{-1} \alpha_{3} e_{3}=X \in S$, and so $X-\left(\alpha_{1} e_{1}+\alpha_{3} e_{3}\right) \in S$. This implies that $-\left(t^{-1}-1\right) \alpha_{3} e_{3} \in S$. Then we get $e_{3} \in S$, this contradicts (a). Thus $\alpha_{1} e_{1}+\alpha_{3} e_{3} \notin S$.

Suppose that $\alpha_{2} e_{2}+\alpha_{3} e_{3} \in S$, with $\alpha_{2} \neq 0$ and $\alpha_{3} \neq 0$. Then $\epsilon_{32}\left(\alpha_{2} e_{2}+\right.$ $\left.\alpha_{3} e_{3}\right)=t \alpha_{2} e_{2}+t^{-1} \alpha_{3} e_{3}+\left((1-t) \alpha_{2}+\left(1-t^{-1}\right) \alpha_{3}\right) e_{4}=X_{1} \in S$ and $\epsilon_{23}\left(\alpha_{2} e_{2}+\alpha_{3} e_{3}\right)=t \alpha_{2} e_{2}+t^{-1} \alpha_{3} e_{3}=Y_{1} \in S$. So $Z_{1}=X_{1}-Y_{1}=\left((1-t) \alpha_{2}+\right.$ $\left.\left(1-t^{-1}\right) \alpha_{3}\right) e_{4} \in S$. On the other hand, $\epsilon_{12}\left(\alpha_{2} e_{2}+\alpha_{3} e_{3}\right)=t^{-1} \alpha_{2} e_{2}+t \alpha_{3} e_{3}+$ $\left(\left(1-t^{-1}\right) \alpha_{2}+(1-t) \alpha_{3}\right) e_{4}=X_{2} \in S$ and $\epsilon_{13}\left(\alpha_{2} e_{2}+\alpha_{3} e_{3}\right)=t^{-1} \alpha_{2} e_{2}+$ $t \alpha_{3} e_{3}=Y_{2} \in S$. And so $Z_{2}=X_{2}-Y_{2}=\left(\left(1-t^{-1}\right) \alpha_{2}+(1-t) \alpha_{3}\right) e_{4} \in S$. Hence, $Z_{1}+Z_{2}=\left(-t-t^{-1}+2\right)\left(\alpha_{2}+\alpha_{3}\right) e_{4} \in S$. But $e_{4} \notin S$ by (a), then $\alpha_{2}+\alpha_{3}=0$, and so $\epsilon_{13}\left(\alpha_{2} e_{2}+\alpha_{3} e_{3}\right)-\epsilon_{31}\left(\alpha_{2} e_{2}+\alpha_{3} e_{3}\right)=\left(t-t^{-1}\right) e_{1} \in S$. This implies that $e_{1} \in S$, which contradicts (a). Thus $\alpha_{2} e_{2}+\alpha_{3} e_{3} \notin S$.

Suppose that $\alpha_{1} e_{1}+\alpha_{4} e_{4} \in S$ with $\alpha_{1} \neq 0$ and $\alpha_{4} \neq 0$. Then $\epsilon_{12}\left(\alpha_{1} e_{1}+\right.$ $\left.\left.\alpha_{4} e_{4}\right)=\alpha_{1} e_{1}+\alpha_{1}\left(t^{-1}-1\right)\right) e_{2}+\alpha_{1}(t-1) e_{3}+\left(\alpha_{1}\left(2-t-t^{-1}\right)+\alpha_{4}\right) e_{4}=X \in S$ and $\epsilon_{32}\left(\alpha_{1} e_{1}+\alpha_{4} e_{4}\right)=\alpha_{1} e_{1}+\alpha_{1}(t-1) e_{2}+\alpha_{1}\left(t^{-1}-1\right) e_{3}+\left(\alpha_{1}(2-t-\right.$ $\left.\left.t^{-1}\right)+\alpha_{4}\right) e_{4}=Y \in S$. So $X-Y=\alpha_{1}\left(-t+t^{-1}\right) e_{2}+\alpha_{1}\left(-t^{-1}+t\right) e_{3} \in S$, which contradicts the previous result. Thus $\alpha_{1} e_{1}+\alpha_{4} e_{4} \notin S$.

Suppose that $\alpha_{2} e_{2}+\alpha_{4} e_{4} \in S$ with $\alpha_{2} \neq 0$ and $\alpha_{4} \neq 0$. Then $\epsilon_{23}\left(\alpha_{2} e_{2}+\right.$ $\left.\alpha_{4} e_{4}\right)-\left(\alpha_{2} e_{2}+\alpha_{4} e_{4}\right)=(t-1) \alpha_{2} e_{2} \in S$, and so $e_{2} \in S$, which contradicts (a). Thus $\alpha_{2} e_{2}+\alpha_{4} e_{4} \notin S$. 
Suppose that $\alpha_{3} e_{3}+\alpha_{4} e_{4} \in S$ with $\alpha_{3} \neq 0$ and $\alpha_{4} \neq 0$. Then $\epsilon_{23}\left(\alpha_{3} e_{3}+\right.$ $\left.\alpha_{4} e_{4}\right)-\left(\alpha_{3} e_{3}+\alpha_{4} e_{4}\right)=\left(t^{-1}-1\right) \alpha_{3} e_{3} \in S$, and so $e_{3} \in S$, which contradicts (a). So $\alpha_{3} e_{3}+\alpha_{4} e_{4} \notin S$.

Therefore, $\alpha_{i} e_{i}+\alpha_{j} e_{j} \notin S$ for any $1 \leq i \neq j \leq 4$.

(c) $\alpha_{i} e_{i}+\alpha_{j} e_{j}+\alpha_{k} e_{k} \notin S$ for any $1 \leq i \neq j \neq k \leq 4$.

Suppose that $\alpha_{1} e_{1}+\alpha_{2} e_{2}+\alpha_{3} e_{3} \in S$ with $\alpha_{1} \neq 0, \alpha_{2} \neq 0$, and $\alpha_{3} \neq 0$. Then $\epsilon_{13}\left(\alpha_{1} e_{1}+\alpha_{2} e_{2}+\alpha_{3} e_{3}\right)-\left(\alpha_{1} e_{1}+\alpha_{2} e_{2}+\alpha_{3} e_{3}\right)=(t-1) \alpha_{2} e_{2}+\left(t^{-1}-1\right) \alpha_{3} e_{3} \in$ $S$, which contradicts (b). So $\alpha_{1} e_{1}+\alpha_{2} e_{2}+\alpha_{3} e_{3} \notin S$.

Suppose that $\alpha_{1} e_{1}+\alpha_{2} e_{2}+\alpha_{4} e_{4} \in S$ with $\alpha_{1} \neq 0, \alpha_{2} \neq 0$, and $\alpha_{4} \neq 0$. Then $\epsilon_{13}\left(\alpha_{1} e_{1}+\alpha_{2} e_{2}+\alpha_{4} e_{4}\right)-\left(\alpha_{1} e_{1}+\alpha_{2} e_{2}+\alpha_{4} e_{4}\right)=(t-1) \alpha_{2} e_{2} \in S$, and so $e_{2} \in S$, which contradicts (a). So $\alpha_{1} e_{1}+\alpha_{2} e_{2}+\alpha_{4} e_{4} \notin S$.

Suppose that $\alpha_{1} e_{1}+\alpha_{3} e_{3}+\alpha_{4} e_{4} \in S$ with $\alpha_{1} \neq 0, \alpha_{3} \neq 0$, and $\alpha_{4} \neq 0$. Then $\epsilon_{13}\left(\alpha_{1} e_{1}+\alpha_{3} e_{3}+\alpha_{4} e_{4}\right)-\left(\alpha_{1} e_{1}+\alpha_{3} e_{3}+\alpha_{4} e_{4}\right)=\left(t^{-1}-1\right) \alpha_{3} e_{3} \in S$, and so $e_{3} \in S$, which contradicts (a). So $\alpha_{1} e_{1}+\alpha_{3} e_{3}+\alpha_{4} e_{4} \notin S$.

Suppose that $\alpha_{2} e_{2}+\alpha_{3} e_{3}+\alpha_{4} e_{4} \in S$ with $\alpha_{2} \neq 0, \alpha_{3} \neq 0$, and $\alpha_{4} \neq 0$. Then $\epsilon_{13}\left(\alpha_{2} e_{2}+\alpha_{3} e_{3}+\alpha_{4} e_{4}\right)-\left(\alpha_{2} e_{2}+\alpha_{3} e_{3}+\alpha_{4} e_{4}\right)=(t-1) \alpha_{2} e_{2}+\left(t^{-1}-1\right) \alpha_{3} e_{3} \in$ $S$, which contradicts (b). So $\alpha_{2} e_{2}+\alpha_{3} e_{3}+\alpha_{4} e_{4} \notin S$.

Therefore, $\alpha_{i} e_{i}+\alpha_{j} e_{j}+\alpha_{k} e_{k} \notin S$ for any $1 \leq i \neq j \neq k \leq 4$.

(d) $\alpha_{1} e_{1}+\alpha_{2} e_{2}+\alpha_{3} e_{3}+\alpha_{4} e_{4} \notin S$.

Suppose that $\alpha_{1} e_{1}+\alpha_{2} e_{2}+\alpha_{3} e_{3}+\alpha_{4} e_{4} \in S$. Then $\epsilon_{13}\left(\alpha_{1} e_{1}+\alpha_{2} e_{2}+\alpha_{3} e_{3}+\right.$ $\left.\alpha_{4} e_{4}\right)-\left(\alpha_{1} e_{1}+\alpha_{2} e_{2}+\alpha_{3} e_{3}+\alpha_{4} e_{4}\right)=(t-1) \alpha_{2} e_{2}+\left(t^{-1}-1\right) \alpha_{3} e_{3} \in S$, which contradicts (b).

Therefore, $\alpha_{1} e_{1}+\alpha_{2} e_{2}+\alpha_{3} e_{3}+\alpha_{4} e_{4} \notin S$.

Thus, $\mathbb{C}^{4}$ contains no nontrivial invariant subspace under $\hat{\phi}_{B}(t) \otimes \hat{\phi}_{B}(m)$. Therefore, $\hat{\phi}_{B}(t) \otimes \hat{\phi}_{B}(m)$ is irreducible.

\section{REFERENCES}

[1] Valerij G. Bardakov, The structure of the group of conjugating automorphisms and the linear representation of the braid groups of some manifolds, Algebra i Logika., 42, No. 5 (2003), 515-541.

[2] Valerij G. Bardakov, Extending representations of braid groups to the automorphism groups of free groups, Journal of Knot Theory and Its Ramifications, 14, No. 8 (2005), 1087-1098.

[3] J. S. Birman, Braids, links and mapping class groups, Annals of Mathematical studies, No. 8, Rrinceton University Press, (1974).

[4] W. Burau, Braids, Uber Zopfgruppen and gleichsinnig verdrillte Verkettungen, Abh. Math. Semin. Hamburg Univ, 11, (1936), 179-186.

[5] J. McCool, On basis conjugating automorphisms of free groups, Can. J. Math., 38, No. 6 (1986), 1525-1529.

[6] A. G. Savushkina, On group of conjugating automorphisms of free group, Matem. Zametki, 60, No. 1 (1996), 92-108 (in Russian).

Mohamad N. Nasser, Department of Mathematics and Computer Science, Beirut Arab University, P.O. Box 11-5020, Beirut, Lebanon

Email address: m.nasser@bau.edu.1b

Mohammad N. Abdulrahim, Department of Mathematics and Computer Science, Beirut Arab University, P.O. Box 11-5020, Beirut, Lebanon

Email address: mna@bau.edu.lb 